\title{
SPECTRAL DISTRIBUTIONS AND THE BREAKING OF ISOSPIN AND SUPERMULTIPLET SYMMETRIES IN NUCLEI
}

\author{
K. T. HECHT and J. P. DRAAYER + \\ Physics Department, The University of Michigan, Ann Arbor, Michigan 48104, USA t†
}

Received 7 January 1974

\begin{abstract}
Knowledge of the Racah algebra for the higher unitary groups is exploited to give a general formula for the partial widths for the direct product subgroup $U(N / k) \times U(k)$ of the full unitary group $U(N)$ of a given shell model vector space. This formula makes it possible to separate the second moments of dynamical operators into the internal and external parts which are needed for detailed applications of the spectral distribution technique. Specific applications are made for $k=2$ (isospin) and $k=4$ (Wigner supermultiplet symmetry). Explicit expressions are given for the isospin breaking contributions to the spectral widths which make it possible to estimate the intensities of isospin impurities in an average state of a given isospin. The goodness of Wigner supermultiplet symmetry is examined for the $2 s-1 \mathrm{~d}$ shell with a detailed example, the $A=25$ nuclel, for which partial widths have been calculated for various modifications of the Kuo-Brown interaction to give a simple measure of the amount of mixing to be expected between states of different space symmetry.
\end{abstract}

\section{Introduction}

Recently French and collaborators ${ }^{1-3}$ ) have shown that spectral distribution methods may prove to be a powerful alternative to the conventional techniques of "microscopic" nuclear spectroscopy with its limitations to shell model vector spaces of manageable dimensions. The usefulness of the spectral distribution method stems from the fact that the low-order moments of dynamical operators are usually the most significant quantities. In estimating, for example, the distribution in energy of the states of some fixed symmetry, it is sufficient to calculate the low-order moments of the Hamiltonian, averaged over the subset of many-particle states belonging to a specific irreducible representation of the relevant symmetry group. The first moments of $H$ give the centroids of the various irreducible representations, while the spectral widths of the irreducible representations are governed by the second moments of $H$. So far the most detailcd applications of spectral distribution techniques to the higher symmetry groups have involved the unitary groups such as $\mathrm{U}(4)$ and $\mathrm{SU}(3)$, particularly for nuclei of the 2 s/ld shell ${ }^{4-7}$ ). Since the average of $H, H^{2}, \ldots$ over an irreducible representation $[f]$ involves a sum of diagonal matrix elements with equal weight for all states of $[f]$, only the scalar (invariant) pieces of $H, H^{2}, \ldots$ can make contributions to such averages. The calculation of such averages is then particularly

$\dagger$ Present address: Physics Dept., University of Rochester, Rochester, New York 14627.

t+ Work supported by the US National Science Foundation. 
simple when the Casimir invariants of the symmetry group in question furnish a sufficient number of invariant operators to reproduce the averages of $H$ and $H^{2}$. This is the case for the direct product subgroup $\mathrm{U}(N / k) \times \mathrm{U}(k)$ for the full unitary group $\mathrm{U}(N)$, where $N$ is the total number of available single-particle states for the shell model space in question, (e.g. $N=24$ for the $2 \mathrm{~s}-1 \mathrm{~d}$ shell). The cases $\mathrm{k}=4$ (Wigner supermultiplet symmetry) and $k=2$ (isospin symmetry) have the greatest physical significance. Parikh ${ }^{6}$ ) has calculated the centroids and widths for all irreducible representations of $U(4)$ and nuclei in the $2 s-1 d$ shell by exploiting the fact that averages of $H$ and $H^{2}$ over an irreducible representation $[f]$ can be expressed solely in terms of particle number and the Casimir invariants of SU(4). Since the widths in general turn out to be comparable to or larger than the spacings between the centroids of different irreducible representations, the goodness of SU(4) symmetry for the $2 \mathrm{~s}-1 \mathrm{~d}$ shell is in question. The simple width to spacing ratios, however, cannot give a reliable measure of symmetry breaking since two overlapping representations can coexist without strong mixing. To gain a measure of the average admixings of different irreducible representations it is important to know what part of the width comes from intermediate states in the irreducible representation $[f]$ itself, and what part from states outside of $[f]$. The detailed applications of the spectral distribution method require a separation of the second moments into such internal and external parts ${ }^{2}$ ). These partial widths can no longer be determined from the group invariants alone but require a knowledge of the Racah algebra of the group. The averaging over states of an irreducible representation, however, eliminates all Wigner coefficients, so that a knowledge of the Racah coefficients for the relevant symmetry group is sufficient to determine the partial widths.

Since many of the symmetrics associated with the vector space of the nuclear shell model are highly approximate, it is important to develop simple a priori tests for the goodness of nuclear symmetries which can give a measure of symmetry breaking to be expected before a detailed decomposition of complicated $n$-particle functions into irreducible representations of a given symmetry group is carried out. In many cases the realistic effective interactions used in shell model calculations have now been fully classified as to their irreducible tensor character under some of the higher symmetry groups ${ }^{8-10}$ ), making it possible to compare the relative strengths of the symmetry breaking and symmetry preserving terms of the Hamiltonian. However, it is not clear how these strengths are best weighted. The most straightforward weighting may give a reliable measure of symmetry breaking only for the two particle system, while it may overestimate the extent of symmetry breaking in systems of larger numbers of particles ${ }^{11}$ ). Since the spectral distribution technique serves to propagate information from systems of small particle number to systems of arbitrary particle number, it is particularly suited to test the goodness of nuclear symmetries in complicated many-particle systems. The symmetry breaking contribution to the width, connecting representation $[f]$ to representation $\left[f^{\prime}\right]$, gives a simple quantitative measure of the amount of symmetry breaking for an average state of $[f]$. The use- 
fulness of such partial widths in estimating mixing intensities has been investigated by Parikh and Wong ${ }^{12}$ ). However, the actual calculation of partial widths to data has been limited to very simple systems for which the shell model matrix diagonalization has been carried out and the detailed shell model wave functions are therefore known. Since it is the aim of the spectral distribution technique to avoid complicated matrix diagonalizations and indeed give spectral information where such diagonalizations are impossible, it is important to be able to calculate partial widths for the higher symmetry groups by simple techniques which do not require knowledge of wave functions.

It is the purpose of this note to show that recent progress in our knowledge of unitary group Racah coefficients has made it possible to give relatively simple expressions for the partial widths for the direct product subgroup $\mathrm{U}(N / k) \times \mathrm{U}(k)$ of the full unitary group $\mathrm{U}(N)$ of a given shell model vector space. The partial width formula is presented in sect, 2 . Some of the details of the derivation are relegated to an appendix since they require considerable group theoretical language. The results, however, can be expressed in terms of a few sums over products of unitary group Racah coefficients. These sums are simple functions of the axial distances associated with the Young tableaux for the irreducible representations [f]. They are tabulated in appendix 2. Applications are made for both the case $k=2$ (isospin) and $k=4$ (Wigner supermultiplet symmetry). Although isospin distributions in nuclei have been treated in great detail by alternate techniques ${ }^{13}$ ), the detailed application of the Racah algebra for the unitary groups makes it possible to give very explicit but general expressions for the isospin breaking contributions to the widths, and these are presented in sect. 3. Although the general partial width formula for $U(N / k) \times U(k)$ should prove useful in many applications of the spectral distribution method ${ }^{2}$ ), the application of greatest interest in the present study involves its use as a simple $a$ priori test for the goodness of higher symmetries in nuclei. As an example it is used to test the goodness of space symmetry (or Wigner supermultiplet) quantum numbers in the $2 \mathrm{~s}-1 \mathrm{~d}$ shell, that is as a test of $\mathrm{U}(6) \times \mathrm{U}(4)$ symmetry. A detailed application is given in sect. 4 to the nine-particle system of the $2 \mathrm{~s}-1 \mathrm{~d}$ shell $(A=25)$ for which partial widths have been calculated for a few of the effective interactions which have been used in successful shell model calculations in the $2 \mathrm{~s}-1 \mathrm{~d}$ shell. These partial widths give a simple measure of the amount of mixing to be expected between states of different space symmetry and can thus be used to decide how (or whether) a shell model matrix for the $A=25$ system can be truncated in terms of space symmctry quantum numbers.

\section{The partial width formula}

The distribution in energy of the states of some fixed symmetry (specific irreducible representation of some symmetry group) is determined mainly by the centroid and the dispersion of the Hamiltonian. The centroid for the irreducible representation 
$[f]$, the average energy expectation value for $[f]$, is given by

$$
E_{\mathrm{c}}([f])=\frac{1}{N_{f}} \sum_{\mu}\left\langle\left[f^{\prime}\right] \mu|H|[f] \mu\right\rangle,
$$

where $N_{f}$ is the total number of states in $[f]$, and where $\mu$ stands for a complete set of subgroup labels which specify the states of $[f]$. The width of the spectral distribution for $[f]$ is related to the dispersion

$$
\sigma^{2}([f])=\frac{1}{N_{f}} \sum_{\mu}\left\langle[f] \mu\left|H^{2}\right|[f] \mu\right\rangle-\left[E_{\mathrm{c}}([f])\right]^{2}
$$

In order to separate the dispersion into an internal (symmetry preserving) and an external (symmetry breaking) part, the matrix elements of $H^{2}$ are split, to separate contributions which arise from matrix elements of $H$ off-diagonal in $[f]$ from those diagonal in $[f]$,

$$
\begin{aligned}
\sigma^{2}([f]) & \equiv \sigma^{2}([f],[f])+\sum_{\left[f^{\prime}\right] \neq[f]} \sigma^{2}\left([f],\left[f^{\prime}\right]\right) \\
= & \frac{1}{N_{f}} \sum_{\mu \bar{\mu}}\langle[f] \mu] H|[f] \bar{\mu}\rangle\langle[f] \bar{\mu}|H|[f] \mu\rangle-\left[E_{\mathrm{c}}([f])\right]^{2} \\
& +\frac{1}{N_{f}} \sum_{\left[f^{\prime}\right] \neq[f]} \sum_{\mu \mu^{\prime}}\left\langle[f] \mu|H|\left[f^{\prime}\right] \mu^{\prime}\right\rangle\left\langle\left[f^{\prime}\right] \mu^{\prime}|H|[f] \mu\right\rangle .
\end{aligned}
$$

The so-called partial widths $\sigma^{2}\left([f],\left[f^{\prime}\right]\right)$ with $\left[f^{\prime}\right] \neq[f]$, can then serve as a measure of symmetry breaking. In particular, the ratio

$$
\chi^{2}\left([f],\left[f^{\prime}\right]\right)=\frac{\sigma^{2}\left([f],\left[f^{\prime}\right]\right)}{\left[E_{\mathrm{c}}([f])-E_{\mathrm{c}}\left(\left[f^{\prime}\right]\right)\right]^{2}}
$$

may provide a quantitative measure of symmetry breaking. In the limit in which $\chi^{2} \ll 1$, (perturbation theory), the ratio $\chi^{2}$ measures the total intensity of the admixture of all states of $\left[f^{\prime}\right]$ into an average state of $[f]$. Even if $\chi^{2}$ is not very small, however, its magnitude can be used to give a qualitative measure of the amount of admixture of $\left[f^{\prime}\right]$ into an average state of $[f]$.

The Racah algebra needed to calculate the partial widths is particularly simple if the symmetry group is a unitary group or a dircet product of unitary groups, such as $\mathrm{U}(\Omega) \times \mathrm{U}(k)$, with $\Omega=N / k$, where $N$ is the full dimension of the singleparticle shell model vector space. In the latter case the representations can be labeled by the symmetry quantum numbers for $\mathrm{U}(\Omega),[f]=\left[f_{1} f_{2} \ldots f_{\Omega}\right]$, with $\sum_{i} f_{i}=n$. The partition number $f_{i}$ specifies the length of the $i$ th row of the Young tableau which characterizes the symmetry of the $n$-particle space wave function for the case $k=4$ or:the space-spin wave function for the case $k=2,\left(f_{i} \leqq k\right)$. The representation [f]] of. $\mathrm{U}(k)$ which characterizes the symmetry of the $n$-particle spin-isospin function 
$(k=4)$ or isospin function $(k=2)$ must be contragredient to $[f]$, that is $[\tilde{f}]$ is obtained from $[f]$ by a row $\leftrightarrow$ column interchange of the Young tableaux. Since $[\tilde{f}]$ is determined by $[f]$ it can be omitted in labelling the $n$-particle states: $|[f] \mu\rangle=$ $|[f] \alpha \beta\rangle$. The subgroup labels $\mu$ have been split into two parts; $\alpha$ stands for a complete set of subgroup labels for $\mathrm{U}(\Omega), \beta$ for a complete set of subgroup labels for $\mathrm{U}(k)$. In eqs. (1) through (3) the sum over subgroup labels is split into separate sums over $\alpha$ and $\beta ;$ and $N_{f}=\operatorname{dim}[f] \times \operatorname{dim}[\tilde{f}]$, where $\operatorname{dim}[f]$ and $\operatorname{dim}[\tilde{f}]$ are the dimensions of the irreducible representations of $U(\Omega)$ and $U(k)$, respectively. [Useful tabulations can be found in the text by Wybourne $\left.{ }^{14}\right)$.]

Since only the scalar [U $(\Omega)$ and $\mathrm{U}(k)$ invariant] pieces of $H, H^{2}$ can make a contribution to the averages of $H, H^{2}$ over an irreducible representation $[f]$ ([ $\left.[\tilde{f}]\right)$, it will be useful to decompose $H$ into irreducible tensor components under these unitary groups. As usual, the Hamiltonian will be expressed in terms of single-particle creation and annihilation operators $a^{+}, a=\left(a^{+}\right)^{+}$. The $\mathrm{U}(\Omega) \times \mathrm{U}(k)$ irreducible tensor character of these operators is given by

$$
\begin{aligned}
& a_{\alpha_{1} \beta_{1}}^{+}=t\left(a^{+}\right)_{\alpha_{1} \beta_{1}}^{[1] 1]}, \\
& a_{\alpha_{1} \beta_{1}}=(-1)^{\eta\left(\alpha_{1}\right)+\eta\left(\beta_{1}\right)} t(a)_{\alpha^{*} \beta^{*} \beta_{1}}^{\left[1^{\Omega-1}\right]\left[1^{k-1}\right]} \text {. }
\end{aligned}
$$

The annihilation operator transforms according to the conjugate representations $\left[1^{\Omega-1}\right]\left[1^{k-1}\right]$, described by Young tableaux of a single column of length $\Omega-1$ and $k-1$, respectively. (Note that in general the conjugate representations $[f]$ and $\left[f^{*}\right]$ of $\mathrm{SU}(\Omega)$ are related by: $\left[f^{*}\right]=\left[f_{1}-f_{\Omega}, f_{1}-f_{\Omega-1}, \ldots, f_{1}-f_{2}, 0\right]$ for $[f]=\left[f_{1} f_{2}\right.$ $\ldots f_{\Omega}$.) The subgroup labels $\alpha^{*}, \beta^{*}$ refer to states in the conjugate subgroup representation. For the single-particle representation, and $\mathrm{U}(\Omega) \times \mathrm{U}(4)$ for example, $\alpha_{1}=l m_{l}$ and $\alpha_{1}^{*}=l,-m_{l}$; while $\beta_{1}=\frac{1}{2} m_{s}, \frac{1}{2} m_{t} ; \beta_{1}^{*}=\frac{1}{2}-m_{s}, \frac{1}{2}-m_{t}$; in terms of the usual orbital, spin, and isospin angular momentum quantum numbers. As always, the phase factor $\eta(\alpha, \beta)$ introduced by the conjugation process is somewhat dependent on phase conventions. Since all final results of this investigation will be independent of such phase factors (appendix 1), no specific choice of phase convention needs to be made. [Note that in coupling operators $a$ it is advantageous to use the irreducible tensor form $t(a)$ of eq. (5).]

Using Wigner coefficients for the groups $\mathrm{U}(\Omega)$ and $\mathrm{U}(k)$, the two-particle operators can be coupled to components of definite irreducible tensor character

$$
\left[\boldsymbol{a}^{+} \times \boldsymbol{a}^{+}\right]_{\alpha_{2} \beta_{2}}^{\left[f_{2}\right]\left[\tilde{f}_{2}\right]},[t(\boldsymbol{a}) \times t(\boldsymbol{a})]_{\alpha_{2}{ }^{*} \tilde{\beta}_{2}{ }^{*}}^{\left.\left[f_{2}^{*}\right] \tilde{f} \tilde{2}^{*}\right]},
$$

with two possible two-particle symmetries: $\left[f_{2}\right]\left[\tilde{f}_{2}\right]=[2]\left[1^{2}\right]$, or $s$, and $\left[f_{2}\right]\left[\tilde{f}_{2}\right]=$ $\left[1^{2}\right][2]$, or $a$, where $s$ and a refer to symmetric and antisymmetric coupling in the subspace of $\mathrm{U}(\Omega)$. The corresponding symmetries for the pair annihilation operators are $\left[f_{2}^{*}\right]\left[\tilde{f}_{2}^{*}\right]=\left[2^{\Omega-1}\right]\left[1^{k-2}\right]$, and $\left[1^{\Omega-2}\right]\left[2^{k-1}\right]$. Finally, a $(1+2)$-body operator can be expressed in terms of the basic irreducible tensor operators $T_{\alpha_{0} \beta_{0}}^{\varphi_{0}\left[f_{0}\left[\tilde{F}_{0}\right]\right.}$ of the type

$$
\begin{gathered}
{\left[a^{+[1][1]} \times t(a)^{\left[1^{\Omega-1}\right]\left[1^{k-1}\right]}\right]_{\alpha_{0} \beta_{0}}^{\left[f_{0}\right]\left[\tilde{F}_{0}\right]},} \\
{\left[\left[\boldsymbol{a}^{+} \times \boldsymbol{a}^{+}\right]^{\left[f_{2}\right]\left[\tilde{f}_{2}\right]} \times[t(a) \times t(a)]^{\left[f_{2}^{*}\right]\left[\tilde{f}^{*}{ }_{2}\right]}\right]_{\alpha_{0} \beta_{0}}^{\left[f_{0}\right]\left[\tilde{F}_{0}\right]} .}
\end{gathered}
$$


For shorthand purposes the symbol $\varphi_{0}$ will be used in place of $\left[f_{2}\right]\left[f_{2}^{\prime *}\right]$ or the analogous one-body operators.

There are then five types of these basic operators, corresponding to the one-body operators with $\varphi_{0}=[1]\left[1^{\Omega-1}\right]$ and the four types of two-body operators corresponding to the four possible couplings, ss, aa, sa, and as, where the pair-creation and annihilation operator symmetries refer to the subspace of $\mathrm{U}(\Omega)$. with $\varphi_{0}=\left[f_{2}\right]\left[f_{2}^{\prime *}\right]=[2]\left[2^{\Omega-1}\right],\left[1^{2}\right]\left[1^{\Omega-2}\right],[2]\left[1^{\Omega-2}\right]$, and $\left[1^{2}\right]\left[2^{\Omega-1}\right]$, respectively. (Note that the redundant $\left[\tilde{f}_{2}\right]\left[\tilde{f}_{2}^{\prime *}\right]$ have been omitted from the labels $\varphi_{0}$.) The coupling for the five types of basic operators is illustrated for the subspace of $\mathrm{U}(\Omega)$
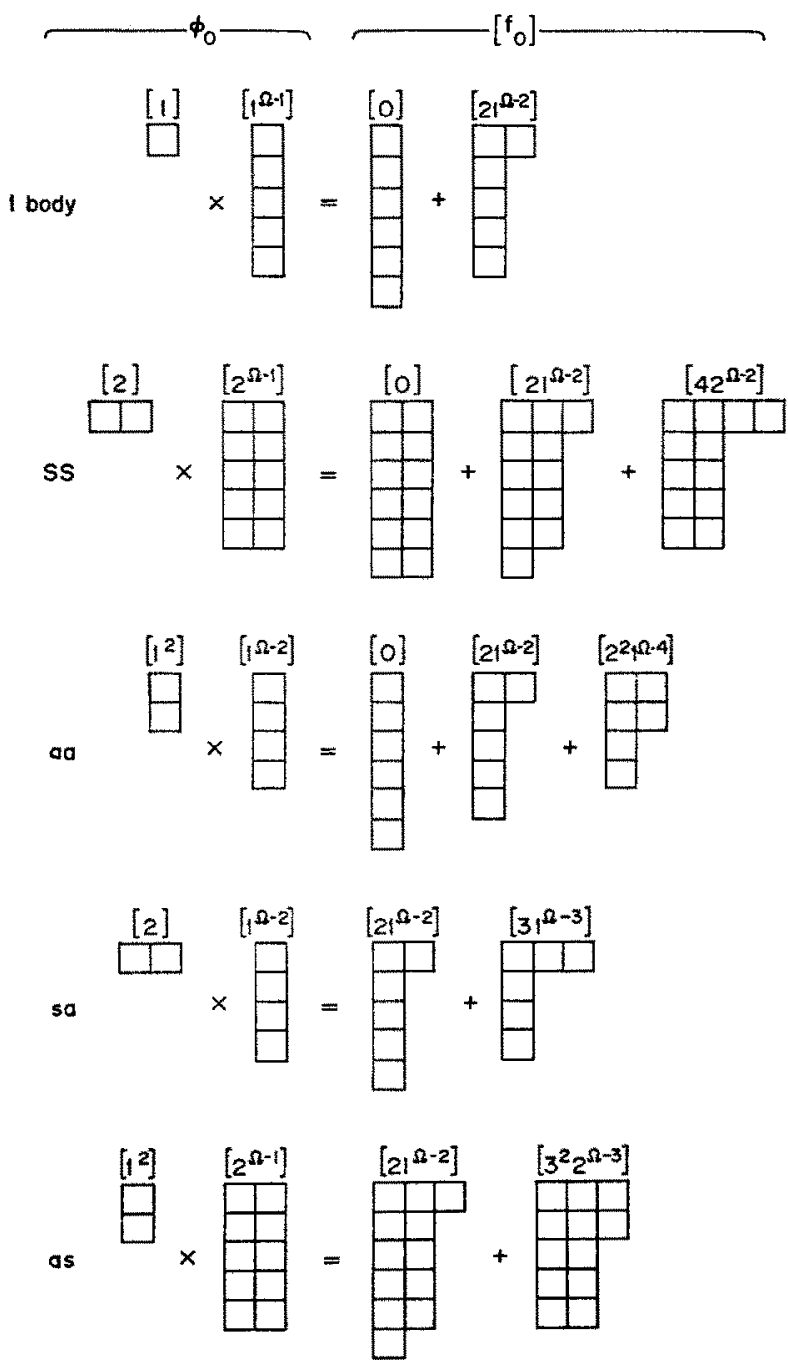

Fig. 1. The basic one- and two-body irreducible tensor components for the unitary group $\mathrm{U}(\Omega)$, illustrated for $\Omega=6$. 
in fig. 1 which shows that there are in all six possible symmetries $\left[f_{0}\right]$. The full irreducible tensor character of the basic operators is obtained by combining these with the corresponding symmetries $\left[\tilde{F}_{0}\right]$ for the subspace of $\mathrm{U}(k)$. With $k=4$, for example, ss tensors (that is, tensors with $\varphi_{0}=[2]\left[2^{\Omega-1}\right]$ ) have $\mathrm{SU}(\Omega) \times \mathrm{SU}(k)$ irreducible tensor character which includes the possibilities $\left[f_{0}\right]\left[\widetilde{F}_{0}\right]=\left[42^{\Omega-2}\right]\left[2^{2}\right],\left[42^{\Omega-2}\right]$ $\left[21^{2}\right],\left[42^{\Omega-2}\right][0] ;\left[21^{\Omega-2}\right]\left[2^{2}\right],\left[21^{\Omega-2}\right]\left[21^{2}\right],\left[21^{\Omega-2}\right][0] ;[0]\left[2^{2}\right],[0]\left[21^{2}\right],[0][0]$. With $\left[f_{2}^{\prime}\right] \neq\left[f_{2}\right]$, the irreducible representations $\left[f_{0}\right]\left[\widetilde{F}_{0}\right]$ are not all self-conjugate; but the full Hamiltonian always contains conjugate partners, such as $\left[3^{2} 2^{\Omega-3}\right]$ and $\left[31^{\Omega-3}\right]$, with equal strength. Note also that $\left[\widetilde{F}_{0}\right]$ is not related by $\left[f_{0}\right]$ by a row $\leftrightarrow$ column interchange. In the above, the invariant components have been denoted by irreducible representation labels $[0]$ for the special unitary groups; e.g. $[0] \equiv\left[2^{\Omega}\right] \equiv$ $\left[1^{\Omega}\right]$ for $\mathrm{SU}(\Omega)$.

The Hamiltonian is expanded in terms of the basic irreducible tensor operators

$$
H=\sum_{\varphi_{0}\left[f_{0}\right]\left[\tilde{F}_{0}\right]} \sum_{\alpha_{0} \beta_{0}} c\left(\varphi_{0}\left[f_{0}\right]\left[\tilde{F}_{0}\right] \alpha_{0} \beta_{0}\right) T_{\alpha_{0} \beta_{0}}^{\varphi_{0}\left[f_{0}\right]\left[\tilde{F}_{0}\right]},
$$

where the strength coefficients, $c$, can be determined for any effective $(1+2)$-body interaction. The centroids, $E_{\mathrm{c}}([f])$, are determined entirely by the components with $\left[f_{0}\right]\left[\tilde{F}_{0}\right]=[0][0]$. The averages of $H^{2}$ are determined by the $\mathrm{U}(\Omega)$ and $\mathrm{U}(k)$ invariant parts of $H^{2}$, which are related only to the intensities of the various components $\left[f_{0}\right]\left[\widetilde{F}_{0}\right]$ of $H$, (involving sums over the subgroup labels $\alpha_{0} \beta_{0}$ ). They are determined by the intensity coefficients ${ }^{\dagger}$

$$
C\left(\varphi_{0} \varphi_{0}^{\prime}\left[f_{0}\right]\left[\tilde{F}_{0}\right]\right)=\sum_{\alpha_{0} \beta_{0}} c\left(\varphi_{0}\left[f_{0}\right]\left[\widetilde{F}_{0}\right] \alpha_{0} \beta_{0}\right) c\left(\varphi_{0}^{\prime}\left[f_{0}\right]\left[\tilde{F}_{0}\right] \alpha_{0} \beta_{0}\right)
$$

Since the notation for the group $\mathrm{U}(\Omega) \times \mathrm{U}(k)$ is somewhat cumbersome, the details of the derivation of the partial width formula will be relegated to appendix 1 . The spirit of the derivation, however, will be illustrated by decomposing the average of a much simpler operator, $\mathcal{O}^{2}$, into partial widths. It will be assumed that $\mathcal{O}$ can be expressed solely in terms of spherical tensor operators; that is, the full symmetry $\mathrm{U}(\Omega) \times \mathrm{U}(k)$ is replaced by a much simpler symmetry corresponding to some group $\mathrm{SU}(2)$ or $\mathrm{R}(3)$. The operator $\mathcal{O}$ is assumed to be hermitian with spherical tensor decomposition

$$
\mathcal{O}=\sum_{\varphi_{0} J_{0} M_{0}} c\left(\varphi_{0} J_{0} M_{0}\right) T_{M_{0}}^{\varphi_{0} J_{0}}=\mathcal{O}^{+}=\sum_{\varphi_{0} J_{0} M_{0}} c\left(\varphi_{0} J_{0} M_{0}\right)(-1)^{M_{0}} T_{-M_{0}}^{\varphi_{0} J_{0}} .
$$

The average of $\mathcal{O}^{2}$ over the states of some irreducible representation $J$ is determined solely by the scalar parts of $\mathcal{O}^{2}$

$$
\left\langle\mathcal{O}^{2}\right\rangle_{J}=\sum_{\varphi_{0} \varphi^{\prime} J_{0} J_{0}} C\left(\varphi_{0} \varphi_{0}^{\prime} J_{0}\right) \frac{(-1)^{J_{0}}}{\left[2 J_{0}+1\right]^{\frac{1}{2}}}\left\langle J\left\|\left[T^{\varphi_{0} J_{0}} \times T^{\varphi^{\prime} J_{0}}\right]^{0}\right\| J\right\rangle,
$$

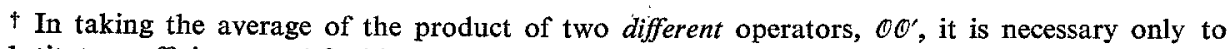
substitute coefficients $c, c^{\prime}$ for the tensor decomposition of the two different operators. 
where

$$
C\left(\varphi_{0} \varphi_{0}^{\prime} J_{0}\right)=\sum_{M_{0}} c\left(\varphi_{0} J_{0} M_{0}\right) c\left(\varphi_{0}^{\prime} J_{0} M_{0}\right)
$$

If the tensors $T^{\varphi_{0} J_{0}},\left(T^{\varphi^{\prime} J_{0} J_{0}}\right)$ are themselves built from simpler operators of spherical tensor rank $J_{2}$ and $J_{2}^{\prime}$, (or $J_{1}$ and $J_{1}^{\prime}$ ), in the sense of the operators $(7)$, the reduced matrix element of eq. (10) can be evaluated by recoupling techniques

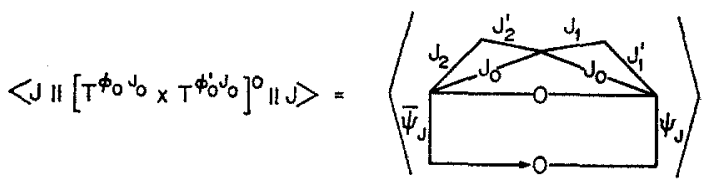

$$
\begin{aligned}
& =\sum_{J^{*}} U\left(J J_{0} J J_{0} ; J^{\prime} O\right) \sum_{J_{2}^{*}} U\left(J J_{2} J^{\prime} J_{2}^{\prime} ; J_{2}^{\prime \prime} J_{0}\right) \sum_{J_{1}^{*}} U\left(J_{1} J_{1}^{\prime} J^{\prime} J ; J_{0} J_{1}^{\prime \prime}\right)
\end{aligned}
$$

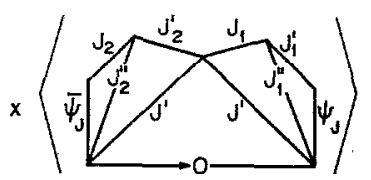

in the pictorial notation of ref. ${ }^{13}$ ); where the $U$-coefficients are ordinary angular momentum recoupling coefficients (Racah coefficients in unitary form). The first recoupling transformation, involving the sum over $J^{\prime}$, gives the decomposition of $\left\langle\mathcal{O}^{2}\right\rangle_{J}$ into partial widths $\sigma^{2}\left(J, J^{\prime}\right)$. The subsequent recoupling transformations, involving sums over $J_{1}^{\prime \prime}$ and $J_{2}^{\prime \prime}$, reduce the evaluation of the matrix elements of $T^{\varphi_{0} J_{0}}$ and $T^{\varphi^{\prime} \mathrm{J}_{0}}$ to the simpler matrix elements of one- or two-particle operators. Specifically, if $\mathcal{O}$ is made up only of one-body operators

$$
\begin{aligned}
\left\langle\mathcal{O}^{2}\right\rangle_{J} & =\sum_{J^{\prime}} \sum_{\varphi_{0} \varphi^{\prime} J_{0}} \frac{C\left(\varphi_{0} \varphi_{0}^{\prime} J_{0}\right)}{\left(2 J_{0}+1\right)} \sum_{J^{\prime \prime}{ }_{2} J^{\prime \prime}} \\
& \times \frac{\left\langle J^{\prime}\left\|a^{+J^{\prime}{ }_{2}}\right\| J_{2}^{\prime \prime}\right\rangle\left\langle J\left\|a^{+J_{2}}\right\| J_{2}^{\prime \prime}\right\rangle\left\langle J^{\prime}\left\|a^{+J_{1}}\right\| J_{1}^{\prime \prime}\right\rangle\left\langle J\left\|a^{+J^{\prime}{ }_{1}}\right\| J_{1}^{\prime \prime}\right\rangle}{\left[\left(2 J_{2}^{\prime}+1\right)\left(2 J_{1}^{\prime}+1\right)\right]^{\frac{1}{2}}} \\
& \times \frac{U\left(J^{\prime} J_{2}^{\prime} J J_{2} ; J_{2}^{\prime \prime} J_{0}\right)}{U\left(J^{\prime} J_{2}^{\prime} J^{\prime} J_{2}^{\prime} ; J_{2}^{\prime \prime} 0\right)} \frac{U\left(J^{\prime} J_{1}^{\prime} J J_{1} ; J_{1}^{\prime \prime} J_{0}\right)}{U\left(J^{\prime} J_{1}^{\prime} J^{\prime} J_{1}^{\prime} ; J_{1}^{\prime \prime} 0\right)},
\end{aligned}
$$

where the $U$-coefficients in the denominators, with $J=0$, are merely convenient ways of expressing simple dimensional and phase factors. In all applications to physical problems, simple groups $\mathrm{SU}(2)$ are always imbedded in higher symmetry groups. Labels $J, J^{\prime}$. . w will be insufficient to specify $n$-particle states, and the reduced matrix elements of $\boldsymbol{a}^{+}$will be complicated functions of additional quantum numbers, expressed in terms of the usual fractional parentage coefficients, so that nothing much is gained by expression (12). However, if the $\mathrm{SU}(2)$ symmetry of this simple example is replaced by the full unitary symmetry of the shell model vector space, or by the group $\mathrm{U}(\Omega) \times \mathrm{U}(k)$; - that is $J \rightarrow[f]$; - then the reduced matrix elements of $a^{+}$, 
(or $\left[\boldsymbol{a}^{+} \times \boldsymbol{a}^{+}\right]$) are simple constants, independent of $[f]$. In particular, $\left\langle\left\|\boldsymbol{a}^{+}\right\|\right\rangle=n^{\frac{1}{2}}$, where $n$ is the number of particles in the state $[f]$ or $\left[f^{\prime}\right]$. The recoupling coefficients above are replaced by Racah coefficients for the higher unitary groups, and the partial widths are reduced to a few simple sums if the Racah algebra for the higher unitary groups has been worked out.

For the direct product subgroup $\mathrm{U}(\Omega) \times \mathrm{U}(k)$ of the full unitary group, the partial width formula, which is the direct generalization of eq. (12), has the form (appendix 1):

$$
\begin{aligned}
& \sigma^{2}\left([f],\left[f^{\prime}\right]\right)=\sum_{\varphi_{0} \varphi^{\prime} 0\left[\tilde{f}_{0}\right]\left[\tilde{F}_{0}\right]} \frac{C\left(\varphi_{0} \varphi_{0}^{\prime}\left[f_{0}\right]\left[\tilde{F}_{0}\right]\right)}{\operatorname{dim}\left[f_{0}\right] \operatorname{dim}\left[\tilde{F}_{0}\right]} \\
& \quad \times \frac{\mathscr{F}(n) \mathscr{F}^{\prime}(n)}{\left[\operatorname{dim}\left[f_{2}^{\prime}\right] \operatorname{dim}\left[\tilde{f}_{2}^{\prime}\right] \operatorname{dim}\left[f_{1}^{\prime}\right] \operatorname{dim}\left[\tilde{f}_{1}^{\prime}\right]\right]^{\frac{1}{2}}} \sum_{\left[f^{\prime \prime} 2\right]\left[f^{\prime \prime} 1\right]} \frac{\mathscr{N}_{\left[f^{\prime \prime}{ }_{2}\right]} \mathscr{N}_{\left[f^{\prime \prime} 1\right]}}{\mathscr{N}_{[f]} \mathscr{N}_{\left[f^{\prime}\right]}} \\
& \quad \times \Sigma\left(\left[f^{\prime}\right]\left[f_{0}\right][f] ;\left[f_{2}^{\prime *}\right]\left[f_{2}\right]\left[f_{2}^{\prime \prime}\right] ;\left[f_{1}^{\prime *}\right]\left[f_{1}\right]\left[f_{1}^{\prime \prime}\right]\right) \\
& \quad \times \tilde{\Sigma}\left(\left[\tilde{f}^{\prime}\right]\left[\tilde{F}_{0}\right][\tilde{f}] ;\left[\tilde{f}_{2}^{\prime *}\right]\left[\tilde{f}_{2}\right]\left[\tilde{f}_{2}^{\prime}\right] ;\left[\tilde{f}_{1}^{\prime *}\right]\left[\tilde{f}_{1}\right]\left[\tilde{f}_{1}^{\prime \prime}\right]\right)
\end{aligned}
$$

where

$$
\begin{aligned}
& \Sigma(\ldots)=\sum_{\rho} \frac{U\left(\left[f^{\prime}\right]\left[f_{2}^{\prime *}\right][f]\left[f_{2}\right] ;\left[f_{2}^{\prime \prime}\right]\left[f_{0}\right] \rho\right)}{U\left(\left[f^{\prime}\right]\left[f_{2}^{\prime *}\right]\left[f^{\prime}\right]\left[f_{2}^{\prime}\right] ;\left[f_{2}^{\prime \prime}\right][0]\right)} \frac{U\left(\left[f^{\prime}\right]\left[f_{1}^{\prime *}\right][f]\left[f_{1}\right] ;\left[f_{1}^{\prime \prime}\right]\left[f_{0}\right] \rho\right)}{U\left(\left[f^{\prime}\right]\left[\tilde{f}_{1}^{\prime *}\right]\left[f^{\prime}\right]\left[f_{1}^{\prime}\right] ;\left[f_{1}^{\prime \prime}\right][0]\right)} \\
& \tilde{\Sigma}(\ldots)=\sum_{\tilde{\rho}} \frac{U\left(\left[\tilde{f}^{\prime}\right]\left[\tilde{f}_{2}^{\prime *}\right][\tilde{f}]\left[\tilde{f}_{2}\right] ;\left[\tilde{f}_{2}^{\prime \prime}\right]\left[\tilde{F}_{0}\right] \tilde{\rho}\right)}{U\left(\left[\tilde{f}^{\prime}\right]\left[\tilde{f}_{2}^{\prime \prime}\right]\left[\tilde{f}^{\prime}\right]\left[\tilde{f}_{2}^{\prime}\right] ;\left[\tilde{f}_{2}^{\prime \prime}\right][0]\right)} \frac{U\left(\left[\tilde{f}^{\prime}\right]\left[\tilde{f}_{1}^{\prime *}\right][\tilde{f}]\left[\tilde{f}_{1}\right] ;\left[\tilde{f}_{1}^{\prime \prime}\right]\left[\tilde{F}_{0}\right] \tilde{\rho}\right)}{U\left(\left[\tilde{f}^{\prime}\right]\left[\tilde{f}_{1}^{\prime *}\right]\left[\tilde{f}^{\prime}\right]\left[\tilde{f}_{1}^{\prime}\right] ;\left[\tilde{f}_{1}^{\prime \prime}\right][0]\right)}
\end{aligned}
$$

Here $\mathscr{F}(n)=n$ if $\varphi_{0}$ denotes a one-body operator, $\mathscr{F}(n)=-n(n-1)$ if $\varphi_{0}$ denotes a two-body operator [as defined in eqs. (7) and (A.1)]; similarly for $\mathscr{F}^{\prime}(n)$ and $\varphi_{0}^{\prime}$. The intensity coefficients, $C$, follow from the tensor decomposition of any given effective Hamiltonian and are given by eqs. (8) and (9). Besides its dependence on the dimension factors, $\operatorname{dim}[f]=$ dimension of the representation $[f]$ of $\mathrm{U}(\Omega), \operatorname{dim}[\tilde{f}]=$ dimension of the representation $[\tilde{f}]$ of $\mathrm{U}(k)$, the partial width formula is now also a function of the dimension factors $\mathscr{N}_{[f]}$, where $\mathscr{N}_{[f]}$ is the dimension of the representation $[f]$ of the symmetric group of degree $n$ (permutation group of $n$ particles). These permutation group dimension factors arise from the separation of the Wigner and Racah algebras of $\mathrm{U}(N)$ into separate Wigner and Racah algebras for $\mathrm{U}(\Omega)$ and $\mathrm{U}(k)$. The final product of Racah coefficients also splits into two factors, the sums $\Sigma$ and $\tilde{\Sigma}$, involving products of Racal coefficients for the groups $\mathrm{U}(\Omega)$ and $\mathrm{U}(k)$, respectively. Now, the $U$-coefficients are Racah coefficients for the higher unitary groups ${ }^{15-18}$ ), written in a notation ${ }^{16-18}$ ) which is a straightforward generalization of that for the ordinary angular momentum recoupling coefficient in unitary form. The $U$-coefficients in the denominators, containing the scalar representation $[f]=[0]$, again are convenicnt ways of writing simple dimensional and phase factors. However, it is now particularly advantageous to express results in terms of such ratios of $U$-coefficients, since final results then become independent of 
specific phase conventions chosen for the unitary group Wigner coefficients (appendix 1). The techniques used to evaluate the higher unitary group Racah coefficients ${ }^{15,19}$ ) also lead to results which are expressed most naturally in terms of such ratios. For the higher unitary groups the coupling and recoupling coefficients are in general functions of multiplicity labels $\rho$. Since the direct product $\left[f^{\prime}\right] \times\left[f_{0}\right]$ is in general not simply reducible, the coupling of representations $\left[f^{\prime}\right]$ and $\left[f_{0}\right]$ can yield a specific representation $[f]$ with a $d$-fold multiplicity, leading to independent coupled states $\left|\left[f^{\prime}\right]\left[f_{0}\right][f] \mu\right\rangle_{\rho}$ with $\rho=1,2, \ldots d$. Although the generalization of the angular mómentum calculus to the higher unitary groups is plagued by this multiplicity problem, Biedenharn, Louck and collaborators ${ }^{15}$ ) have shown that there is a canonical resolution to the multiplicity problem. In their view the unitary group Wigner and Racah coefficients are uniquely defined (with no arbitrariness in the choice of $\rho$ ). The averaging over states of an irreducible representation, however, not only eliminates all Wigner coefficients but also all details of the multiplicity structure. In the partial width formula the dependence on the multiplicity labels $\rho$ survives only in a sum over $\rho$ of a product of unitary group $U$-coefficients (appendix 2). This sum is completely independent of any specific choices made for the multiplicity label (they can be chosen "canonically" or in any arbitrary manner). The resultant sum is a function only of the irreducible representation labels $f_{i}$. [Note that in the present application there is such a multiplicity labeling in only one of the four couplings which make up the recoupling transformation implied by the $U$-coefficients above, since the direct products $[f] \times\left[f_{2}\right]$ are simply reducible in the special case when $\left[f_{2}\right]$ is any two- (or one-) particle or two- (or one-) hole representation.]

The sum over multiplicity labels $\rho$ reduces the quantities $\Sigma$ and $\tilde{\Sigma}$ to simple functions ${ }^{19}$ ) of the symmetry quantum numbers $f_{1}, \ldots, f_{\Omega}$, (or $\tilde{f}_{1}, \ldots, \tilde{f}_{k}$ ) which are determined solely by the axial distances for the Young tableaux describing $[f]$, (or $[\tilde{f}])$. In many cases, with $\left[f^{\prime}\right] \neq[f]$, the $\rho$-sums in the numerator of $\Sigma$ collapse to the trivial value unity, and $\Sigma$ is then given simply by the squares of the Racah coefficients in the denominator and hence by trivial dimension factors [see eq. (A.14)]. As a specific example, let $\left[f^{\prime}\right]$ be related to $[f]$ in the following manner: After adding two squares to every one of the $\Omega$-rows of the Young tableau $[f]$ for $\mathrm{U}(\Omega)$, the tableau for $\left[f^{\prime}\right]$ is obtained by then removing four squares from the row labeled $a$, one square from row $b$, one square from row $c$, two squares from $d, e, \ldots$ until two squares have been removed from $\Omega-3$ different rows, where $a, b, c, d, \ldots$ stand for any of the numbers $1,2,3, \ldots \Omega$, provided $a \neq b \neq c \neq \ldots$. In this example $\left[f^{\prime}\right]$ will be described by the notation

$$
\left[f^{\prime}\right]=\left[f\left(a^{4} b c d^{2} e^{2} \ldots\right)\right]
$$

For $\operatorname{SU}(\Omega)$ this $\left[f^{\prime}\right]$ is equivalent to the one obtained by first adding one square to every row of $[f]$ and subsequently removing three squares from the row labeled $a$, none from rows $b$ and $c$, and one each from rows $d, e, \ldots$; i.e.

$$
\left[f^{t}\right]=\left[f\left(a^{4} b c d^{2} e^{2} \ldots\right)\right]=\left[f\left(a^{3} d e \ldots\right)\right]
$$


corresponding to the cases where $\left[f^{\prime}\right]$ is obtained from $[f]$ by the removal of $2 \Omega$ or $\Omega$ squares, respectively. The representations $[f]$ and $\left[f^{\prime}\right]$ are connected by operators of symmetry $\left[f_{0}\right]$, where $\left[f_{0}\right]$ is made up of $2 \Omega$ and $\Omega$ squares in the two cases. In the specific example above, the representations $[f]$ and $\left[f^{\prime}\right]$ can be connected only by operators of irreducible tensor character $\left[f_{0}\right]=\left[42^{\Omega-2}\right]$, with $\varphi_{0}=[2]\left[2^{2-1}\right]$; or $\left[f_{0}\right]=\left[31^{\Omega-3}\right]$, with $\varphi_{0}=[2]\left[1^{2-2}\right]$. Both for $\left[42^{2-2}\right]$ and for $\left[31^{2-3}\right]$ the representation $\left[f^{\prime \prime}\right]$ is restricted to the single possibility: $\left[f^{\prime \prime}\right]=[f(a a)]$, that is, two squares must be removed from row $a$ in the first step of the recoupling process which connects $[f]$ to $\left[f^{\prime}\right]=\left[f\left(a^{4} b c d^{2} e^{2} \ldots\right)\right]$. In either case the Racah coefficient describes a onedimensional unitary matrix, and its square thus has the trivial value unity. In terms of the above notation the only nontrivial connections, with $\left[f^{\prime}\right] \neq[f]$, involve the representations of the type

$$
\left[f^{\prime}\right]=\left[f\left(a^{3} b c^{2} d^{2} e^{2} \ldots\right)\right]=\left[f\left(a^{2} c d e . .\right)\right] .
$$

In this case the representations $\left[f^{\prime}\right]$ and $[f]$ can be connected by operators of irreducible tensor character $\left[f_{0}\right]=\left[42^{\Omega-2}\right],\left[2^{2} 1^{\Omega-4}\right],\left[3^{2} 2^{\Omega-3}\right],\left[31^{\Omega-3}\right]$, and $\left[21^{\Omega-2}\right]$. The functions $\Sigma$ (or $\tilde{\Sigma}$ ) for this case are tabulated in appendix 2 .

The partial width formula involves a sum over all possible symmetry components $\varphi_{0} \varphi_{0}^{\prime}\left[f_{0}\right]\left[\tilde{F}_{0}\right]$. In general, however, the irreducible tensor character $\left[f_{0}\right]\left[\tilde{F}_{0}\right]$ determines the operator type $\varphi_{0}$. Thus $\varphi_{0}$ and $\varphi_{0}^{\prime}$ are both restricted to the single possibility $[2]\left[2^{\Omega-1}\right]$ if $\left[f_{0}\right]=\left[42^{\Omega-2}\right]$, for example. There is only one combination of symmetries $\left[f_{0}\right]$ and $\left[\widetilde{F}_{0}\right]$, which can connect states $[f]$ to $\left[f^{\prime}\right] \neq[f]$, for which the quantities $\Sigma$ and $\tilde{\Sigma}$ will contain cross terms $\varphi_{0}^{\prime} \neq \varphi_{0}$, namely the combination $\left[f_{0}\right]\left[\widetilde{F}_{0}\right]=\left[21^{\Omega-2}\right]\left[21^{k-2}\right]$. For such irreducible tensors the quantities $\Sigma$ and $\tilde{\Sigma}$ will contain cross tcrms between the four possible types of two-body operators (see fig. 1) and between the four types of two-body operators and one-body operators. For the special case $\left[f^{\prime}\right]=\left[f\left(a^{3} b c^{2} d^{2} e^{2} \ldots\right)\right]$, however, the $\Sigma$ for such cross terms are simply related to the $\Sigma$ for which $\left[f_{2}\right]=\left[f_{1}\right],\left[f_{2}^{\prime}\right]=\left[f_{1}^{\prime}\right]$; [see eqs. (A.18) and (A.19) of appendix 2].

\section{Isospin admixing}

Isospin distributions have been discussed in detail ${ }^{12}$ ) without the use of unitary group Racah algebra. Centroids and spectral widths have been calculated for both overall isospin and isospin configuration distributions by the use of the linear trace equivalents of $H$ and $H^{2}$, where these carry the information contained in the invariant parts of these operators. The detailed application of the Racah algebra for $\mathrm{U}(\Omega) \times$ $\mathrm{U}(2)$, however, now makes it possible to give very explicit expressions for the partial widths, particularly the isospin breaking contributions to the widths connecting states $T$ to $T^{\prime} \neq T$. If these are compared to the centroid separations they give a measure of the amount of admixing of states $T^{t}$ into an average state of $T$. Since the formalism is that of discrete spectroscopy, however, and is therefore restricted to finite-dimensional 
shell model vector spaces involving only bound state single-particle orbits, such admixture coefficients give a measure only of the "internal" admixings (in the language commonly used to discuss isospin impurities). This restriction must be kept in mind since the "external" admixings involving scattering or continuum states are often of greater interest.

For the group $\mathrm{U}(\Omega) \times \mathrm{U}(2)$ the partial width formula eq. (13), takes a particularly simple form. The irreducible representations, $[f]$ and $[\tilde{f}]$ are specified by $n$ and $T$ : $[f]=\left[2^{\frac{1}{2} n-T} 1^{2 T}\right] ;[\tilde{f}]=\left[\tilde{f}_{1} \tilde{f}_{2}\right]=\left[\frac{1}{2} n+T, \frac{1}{2} n-T\right]$. The quantities $\tilde{\Sigma}$ are given in terms of ordinary angular momentum (isospin) recoupling coefficients. The quantities $\Sigma$, which are expressed in terms of simple functions of the axial distances for the Young tableaux for $[f]$ in appendix 2, can now be written in terms of simple functions of $n, T$, and $\Omega$. As a specific example, the sum $\Sigma$ for $[f]=\left[2^{\frac{1}{2} n-T} 1^{2 T}\right],\left[f^{\prime}\right]=$ $\left[2^{\frac{1}{2 n-T-1}} 1^{2 T+2}\right]$ (i.e. $\left.T^{\prime}=T+1\right),\left[f_{0}\right]=\left[2^{2} 1^{\Omega-4}\right] ;\left[f_{2}\right]=\left[f_{1}\right]=\left[1^{2}\right],\left[f_{2}^{\prime *}\right]=$ $\left[f_{1}^{\prime *}\right]=\left[1^{\Omega-2}\right]$, and $\left[f_{2}^{\prime \prime}\right]=\left[f_{1}^{\prime \prime}\right]=\left[2^{\frac{1}{2 n-T-1}} 1^{2 T}\right]$ (i.e. $T_{2}^{\prime \prime}=T_{1}^{\prime \prime}=T$ ) has the simple value

$$
\Omega(\Omega-1) \frac{T(2 T+3)\left(\Omega-\frac{1}{2} n-T\right)}{(T+1)(2 T+1)\left(\frac{1}{2} n+T+2\right)}\left\{\frac{(T+1)\left(\Omega-\frac{1}{2} n-T+1\right)}{2 T\left(\frac{1}{2} n+T+1\right)}-\frac{1}{(\Omega-2)}\right\} .
$$

The sums over $\left[f_{1}^{\prime \prime}\right]\left[f_{2}^{\prime \prime}\right],\left(T_{1}^{\prime \prime}, T_{2}^{\prime \prime}\right)$, involving a few simple functions of this kind can easily be carried out, so that the isospin partial widths can be given as functions of $n, T$, and $\Omega$, in terms of the intensity coefficients $C\left(\varphi_{0} \varphi_{0}^{\prime}\left[f_{0}\right]\left[\tilde{F}_{0}\right]\right)$ for the symmetry breaking part of the interaction, $H$. (For the isospin case it is more natural to use the spherical tensor label $T_{0}$ in place of $\left[\widetilde{F}_{0}\right]$, with $T_{0}=2$ or 1 for $\left[\widetilde{F}_{0}\right]=[4]$ or [2].) If the isospin breaking part of $H$ arises solely from the Coulomb interaction, the only operator types which can make contributions to the partial widths are those with (i) $\varphi_{0}=[1]\left[1^{\Omega-1}\right]$, effective one-body operators which give the contribution due to interactions of the valence particles with the core, and (ii) $\varphi_{0}=\left[1^{2}\right]\left[1^{2-2}\right]$ or $\varphi_{0}=$ aa (see fig. 1), whose strengths are determined by two-particle matrix elements in $T=1$ states. Note that the most general charge-dependent interaction can make contributions through operators of the type $\varphi_{0}=$ sa and as; but, under the assumption that two-particle matrix elements connecting $T=1$ states to $T=0$ states are negligible compared with the $T=1$ Coulomb matrix elements, such terms can be neglected; and only operators with $\varphi_{0}=$ one-body, $\left[f_{0}\right]=\left[21^{\Omega-2}\right], T_{0}=1$; or $\varphi_{0}=\mathrm{aa},\left[f_{0}\right]=\left[21^{\Omega-2}\right], T_{0}=1$ or 2 , or $\left[f_{0}\right]=\left[2^{2} 1^{\Omega-4}\right], T_{0}=1$ or 2 , can make contributions to the partial widths, connecting states $T$ to $T^{\prime}$. In terms of the intensity coefficients for these components of the interaction, the partial width formulae are

$$
\begin{aligned}
& \sigma^{2}\left(T, T^{\prime}=T+1\right)=\frac{2 T+3}{2 T+1} \frac{\left(\Omega-\frac{1}{2} n-T\right)\left(\frac{1}{2} n-T\right)}{3(\Omega-1)(\Omega+1)} \\
& \quad \times\left\{C\left(1 \text {-body, 1-body }\left[21^{\Omega-2}\right] T_{0}=1\right)+\frac{n-2}{\Omega-2} C\left(\text { aa, aa }\left[21^{\Omega-2}\right] 1\right)\right.
\end{aligned}
$$




$$
\begin{gathered}
-\frac{2(n-2)}{[\Omega-2]^{\frac{1}{2}}} C\left(1 \text {-body, aa }\left[21^{\Omega-2}\right] 1\right)+\frac{12 T(T+2)}{5(\Omega-2)} C\left(\mathrm{aa}, \mathrm{aa}\left[21^{\Omega-2}\right] 2\right) \\
+\frac{4(\Omega-1)}{\Omega^{2}(\Omega-3)} C\left(\mathrm{aa}, \mathrm{aa}\left[2^{2} 1^{\Omega-4}\right] 1\right)\left[\frac{(\Omega+2)(n-2)(2 \Omega-n-2)}{4(\Omega-2)}-T(T+2)\right] \\
\left.+\frac{12(\Omega-1)}{5 \Omega^{2}(\Omega-3)}\left(1-\delta_{T 0}\right) C\left(\mathrm{aa}, \mathrm{aa}\left[2^{2} 1^{\Omega-4}\right] 2\right)\left[\frac{(n+2)(2 \Omega-n+2)}{4}-\frac{(\Omega+2) T(T+2)}{(\Omega-2)}\right]\right\}, \\
\sigma^{2}\left(T, T^{\prime}=T+2\right)=C\left(\mathrm{aa}, \mathrm{aa}\left[2^{2} 1^{\Omega-4}\right] T_{0}=2\right) \\
\times \frac{4(2 T+5)\left(\frac{1}{2} n-T\right)\left(\frac{1}{2} n-T-1\right)\left(\Omega-\frac{1}{2} n-T\right)\left(\Omega-\frac{1}{2} n-T-1\right)}{5(2 T+1) \Omega^{2}(\Omega-3)(\Omega+1)}
\end{gathered}
$$

Partial widths $\sigma^{2}\left(T^{\prime}, T\right)$ and $\sigma^{2}\left(T, T^{\prime}\right)$ are related by simple dimensional factors, [cf. eq. (3)]. Specifically

$$
\begin{aligned}
\frac{\sigma^{2}(T+1, T)}{\sigma^{2}(T, T+1)}=\frac{2 T+1}{2 T+3} \frac{\operatorname{dim}\left[2^{\frac{1}{2} n-T} 1^{2 T}\right]}{\operatorname{dim}\left[2^{\frac{1}{2} n-T-1} 1^{2(T+1)}\right]} \\
=\frac{(2 T+1)^{2}\left(\Omega-\frac{1}{2} n+T+2\right)\left(\frac{1}{2} n+T+2\right)}{(2 T+3)^{2}\left(\Omega-\frac{1}{2} n-T\right)\left(\frac{1}{2} n-T\right)} .
\end{aligned}
$$

Similarly,

$$
\frac{\sigma^{2}(T+2, T)}{\sigma^{2}(T, T+2)}=\frac{(2 T+1)^{2}\left(\frac{1}{2} n+T+2\right)\left(\frac{1}{2} n+T+3\right)\left(\Omega-\frac{1}{2} n+T+3\right)\left(\Omega-\frac{1}{2} n+T+2\right)}{(2 T+5)^{2}\left(\frac{1}{2} n-T\right)\left(\frac{1}{2} n-T-1\right)\left(\Omega-\frac{1}{2} n-T\right)\left(\Omega-\frac{1}{2} n-T-1\right)} .
$$

The intensity coefficients, $C$, are determined in terms of the effective single-particle energies, $\varepsilon_{s}$, for protons and neutrons in the orbit $j_{s}$, with degeneracy $\Omega_{s}=\left(2 j_{s}+1\right)$, and in terms of two-particle matrix elements between proton states. In the notation

$$
\begin{gathered}
\Delta \varepsilon_{s}=\frac{1}{2}\left[\left(\varepsilon_{s}\right)_{\text {proton }}-\left(\varepsilon_{s}\right)_{\text {neutron }}\right], \\
\Omega=\sum_{s} \Omega_{s}=\sum_{s}\left(2 j_{s}+1\right), \\
W_{r s t u J}=\left\langle\left[j_{r} j_{s}\right]_{\mathrm{a}} J M_{J} T=1 M_{T}=-1|V|\left[j_{t} j_{u}\right]_{\mathrm{a}} J M_{J} T=1 M_{T}=-1\right\rangle,
\end{gathered}
$$

where $\left[j_{r} j_{s}\right]_{a} J M_{J}$ denotes a normalized angular-momentum-coupled state, antisymmetric in orbital-spin space; the intensity coefficients for a configuration $\left(j_{1} j_{2} \ldots j_{s}\right.$ $\ldots)^{n, T}$ are

$C\left(1-\right.$ body, 1 -body $\left.\left[21^{\Omega-2}\right] T_{0}=1\right)=2 \sum_{s}\left(\Delta \varepsilon_{s}\right)^{2} \Omega_{s_{-}}\left(1-\frac{\Omega_{s}}{\Omega}\right)-4 \sum_{r<s} \Delta \varepsilon_{r} \Delta \varepsilon_{s} \frac{\Omega_{r} \Omega_{s}}{\Omega}$, 


$$
\begin{aligned}
& C\left(\mathrm{aa}, \mathrm{aa}\left[21^{\Omega-2}\right] 1\right)=3 C\left(\mathrm{aa}, \mathrm{aa}\left[21^{\Omega-2}\right] 2\right)=\sum_{J J^{\prime}} \frac{(2 J+1)\left(2 J^{t}+1\right)}{2(\Omega-2)} \\
& \times\left\{\sum_{s} W_{s s s s} W_{s s s, I^{\prime}}\left(\frac{1}{\Omega_{s}}-\frac{1}{\Omega}\right)+\sum_{r<s} W_{r s r s . J} W_{r s r s . I^{\prime}}\left(\frac{\Omega_{r}+\Omega_{s}}{4 \Omega_{r} \Omega_{s}}-\frac{1}{\Omega}\right)\right. \\
& -\frac{2}{\Omega} \sum_{r<s} W_{r r r r} W_{\text {ssss } J^{x}}+2 \sum_{r} \sum_{s \neq r} W_{r r r J} W_{r s r s} \cdot\left(\frac{1}{2 \Omega_{r}}-\frac{1}{\Omega}\right) \\
& -\frac{2}{\Omega} \sum_{\substack{r \\
s \neq r}} \sum_{\substack{s<t \\
t \neq r}} W_{r r r r} W_{s t s t I^{\prime}}-\frac{2}{\Omega} \sum_{\substack{r<s \\
t \neq r, s}} \sum_{\substack{t<u \\
u \neq r, s}} W_{r s r s J} W_{t u t u J^{\prime}} \\
& \left.+\sum_{\substack{r<s \\
t \neq t \neq t}} \sum_{\substack{t \neq s \\
t \neq s+s}}\left[W_{t t r t J^{\prime}}\left(\frac{1}{4 \Omega_{r}}-\frac{1}{\Omega}\right)+W_{t s t s J^{\prime}}\left(\frac{1}{4 \Omega_{s}}-\frac{1}{\Omega}\right)\right]\right\} \\
& -\sum_{s} \sum_{r \neq s} \Delta \varepsilon_{s} W_{r r r J} \frac{\Omega_{s}}{\Omega}+\sum_{s} \sum_{r \neq s} \Delta \varepsilon_{s} W_{s r s r}\left(\frac{1}{2}-\frac{\Omega_{s}}{\Omega}\right) \\
& \left.-\sum_{\substack{s \\
s \neq s}} \sum_{\substack{r<t \\
t \neq s}} \Delta \varepsilon_{s} W_{r t r t} \frac{\Omega_{s}}{\Omega}\right\},
\end{aligned}
$$

$$
\begin{aligned}
& C\left(\mathrm{aa}, \mathrm{aa}\left[2^{2} 1^{2-4}\right] T_{0}=1\right)=3 C\left(\mathrm{aa}, \mathrm{aa}\left[2^{2} 1^{2-4}\right] T_{0}=2\right) \\
& =\frac{1}{8} \sum_{J}(2 J+1)\left[\sum_{r} W_{r r r r}^{2}+\sum_{r<s} W_{r s r s J}^{2}\right] \\
& +\frac{1}{4} \sum_{J J^{\prime}} \frac{(2 J+1)\left(2 J^{\prime}+1\right)}{\Omega-2}\left\{\sum_{r} W_{r r r J} W_{r r r J^{\prime}}\left(\frac{1}{\Omega-1}-\frac{2}{\Omega_{r}}\right)\right. \\
& +\sum_{r<s} W_{r s r s J} W_{r s r s J^{\prime}}\left(\frac{1}{\Omega-1}-\frac{\Omega_{r}+\Omega_{s}}{2 \Omega_{r} \Omega_{s}}\right)+\frac{2}{\Omega-1} \sum_{r<s} W_{r r r J} W_{s s s J^{\prime}} \\
& +2 \sum_{r} \sum_{s \neq r} W_{p r y Y} W_{r s p s J^{\prime}}\left(\frac{1}{\Omega-1}-\frac{1}{\Omega}\right)+\frac{2}{\Omega-1} \sum_{\substack{r \\
s \neq r}} \sum_{\substack{s<t \\
s \neq r}} W_{r r r r J} W_{s t s t J^{*}} \\
& +2 \sum_{\substack{r<s \\
t \neq t^{\prime} r}} \sum_{\substack{t \\
t \neq s}} W_{r s t s J}\left[W_{r t r t I^{\prime}}\left(\frac{1}{\Omega-1}-\frac{1}{2 \Omega_{r}}\right)+W_{s t s t J^{\prime}}\left(\frac{1}{\Omega-1}-\frac{1}{2 \Omega_{s}}\right)\right]
\end{aligned}
$$

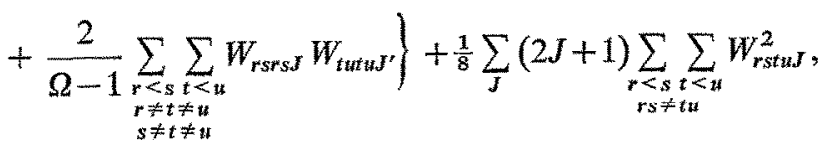


where these follow from a decomposition of the interaction into irreducible tensor components for $\mathrm{U}(\Omega) \times \mathrm{U}(2)$ [see, e.g. ref. $\left.{ }^{3}\right)$ ]. (For the most general charge-dependent interaction the proton-proton matrix elements $V_{\mathrm{pp}}$ of eq. (20) must be replaced by $V_{\mathrm{pp}}-V_{\mathrm{nn}}$ for $T_{0}=1$ tensors, and by $V_{\mathrm{pp}}+V_{\mathrm{nn}}-2 V_{\mathrm{np}}$ for $T_{\mathrm{o}}=2$ tensors.)

For the single shell case, $j^{n}$, only two-body terms of irreducible tensor character $\left[2^{2} 1^{\Omega-4}\right]$ survive. In this simple case

$$
\begin{aligned}
& C\left(\text { aа, аa }\left[2^{2} 1^{\Omega-4}\right] 1\right)=3 C\left(\mathrm{aa}, \mathrm{aa}\left[2^{2} 1^{\Omega-4}\right] 2\right) \\
& \quad=\frac{1}{8}\left\{\sum_{J \text { even }}(2 J+1) W_{J}^{2}-\frac{1}{j(2 j+1)} \sum_{\substack{J^{\prime} \\
\text { even }}}(2 J+1)\left(2 J^{\prime}+1\right) W_{J} W_{J^{\prime}}\right\} .
\end{aligned}
$$

For the $1 \mathrm{f}_{\frac{7}{2}}$ shell, for example, the two-body Coulomb matrix elements $W_{J}$ have been estimated by Jänecke ${ }^{20}$ ) from the experimentally observed Coulomb displacement energies. To within the experimental uncertainties, the matrix elements $W_{J}$ with $J \neq 0$ can be replaced by $\bar{W}_{2}$, the average seniority-two Coulomb matrix elcment. In this approximation the above intensity coefficient is given by

$$
C\left(\mathrm{aa}, \mathrm{aa}\left[2^{2} 1^{2-4}\right] 1\right)=\left(W_{0}-\bar{W}_{2}\right)^{2} \frac{(2 j-1)(j+1)}{8 j(2 j+1)} .
$$

Jänecke's $1 \mathrm{f}_{\frac{7}{2}}$ shell analysis $\left.{ }^{20}\right)$ gives $\left(W_{0}-\bar{W}_{2}\right)_{\exp } \approx 75 \mathrm{keV}$. Estimates for the centroid separation for the $1 \mathrm{f}_{\frac{7}{2}}$ shell have been made by French $\left.{ }^{13}\right) ; E_{\mathrm{c}}\left(T^{\prime}\right)-E_{\mathrm{c}}(T)=$ $0.80 \mathrm{MeV}\left[T^{\prime}\left(T^{\prime}+1\right)-T(T+1)\right]$; and isospin admixture coefficients $\chi^{2}\left(T, T^{\prime}\right)$, as defined in eq. (4), can easily be calculated. For the single shell case the largest admixtures are predicted for the half-full shell and $T=0$. Even in this case $(n=8$, $T=0, T^{\prime}=1$ ), the coefficient $\chi^{2}$ is extremely small, $\chi^{2} \approx 9 \times 10^{-5}$, corresponding to a $T=1$ admixture of only $0.009 \%$ into an average $T=0$ state of ${ }^{48} \mathrm{Cr}$, (based on a pure $1 \mathrm{f}_{\frac{7}{2}}$ model without core excitations). This result confirms conclusions drawn from detailed shell model calculations on the isospin purity of low-lying states in light nuclei ${ }^{21}$ ). Due to the long range nature of the Coulomb potential the major contributions of the Coulomb interaction to the isospin impurities do not come from specifically two-body effects of this two-body interaction but through the average Coulomb potential exerted on a valence proton by the core protons. In the framework of the present description, such effects are determined by the single-particle parameters $\Delta \varepsilon_{s}$ of eq. (18). For nuclei in the $2 \mathrm{~s}-1 \mathrm{~d}$ shell, with $\left.\left(\varepsilon_{\mathrm{s}_{\frac{1}{2}}}-\varepsilon_{\mathrm{d}}\right)_{\mathrm{s}}\right)_{\text {proton }}=495 \mathrm{keV}$ (using the observed ground to first excitcd state separation in ${ }^{17} \mathrm{~F}$ ), and $\left(\varepsilon_{\mathrm{s}_{\frac{1}{2}}}-\varepsilon_{\mathrm{ds}}\right)_{\text {neutron }}=$ $871 \mathrm{keV}$ (from ${ }^{17} \mathrm{O}$ ), and with the assumption that $\left(\varepsilon_{\mathrm{d} \frac{3}{2}}-\varepsilon_{\mathrm{d} \frac{5}{2}}\right)$ are the same for protons and neutrons (the experimental evidence is incomplete), the one-body intensity coefficient, $C$, eq. (21), has the value $0.118 \mathrm{MeV}^{2}$. With the further assumption that two-body effects are completely negligible, the admixture coefficient for ${ }^{28} \mathrm{Si}\left(n=12, T=0, T^{\prime}=1\right)$ is $\chi^{2}(0,1)=0.0074$. [Here a centroid separation, $E_{\mathrm{c}}(1)-E_{\mathrm{c}}(0)$, of $2 \mathrm{MeV}$ has been used; this is an average value for various effective interactions used successfully in the $2 \mathrm{~s}-5 \mathrm{~d}$ shell ${ }^{4}$ ).] The predicted $T=1$ admixture 
into an average $T=0$ state of ${ }^{28} \mathrm{Si}$ (the half-full shell nucleus) is thus $0.74 \%$, which is orders of magnitude larger than the impurities due to the specifically two-body effects of the Coulomb interaction.

The partial width formula can also be used to calculate the internal parts of the widths or the full spectral widths for isospin distributions. [Needed sums over $U$ coefficients are tabulated in ref. $\left.{ }^{19}\right)$.] For a purely charge-independent interaction, $\left(T_{0}=0\right)$, the spectral widths for the configuration $\left(j_{1} j_{2} \ldots j_{s} \ldots\right)^{n, T}$ are given by

$$
\begin{aligned}
& \sigma^{2}(T, T)=\frac{8 C\left(\mathrm{aa}, \mathrm{aa}\left[2^{2} 1^{\Omega-4}\right] T_{0}=0\right)}{3 \Omega^{2}(\Omega+1)(\Omega-1)(\Omega-3)(\Omega-2)}\left\{\frac{1}{2} T^{2}(T+1)^{2}\left(3 \Omega^{2}-7 \Omega+6\right)\right. \\
& +\frac{3}{8} n(n-2)\left(\Omega-\frac{1}{2} n\right)\left(\Omega-\frac{1}{2} n-1\right)(\Omega+1)(\Omega+2) \\
& \left.+T(T+1)(5 \Omega-3)(\Omega+2) \frac{1}{2} n\left(\frac{1}{2} n-\Omega\right)+\frac{1}{2} T(T+1) \Omega(\Omega-1)(\Omega+1)(\Omega+6)\right\} \\
& +\frac{C\left(\mathrm{ss}, \mathrm{ss}\left[42^{2-2}\right] 0\right)}{\Omega^{2}(\Omega+1)(\Omega-1)}\left\{[n(n+2)-4 T(T+1)]\left[\left(\Omega-\frac{1}{2} n\right)\left(\Omega-\frac{1}{2} n+1\right)-T(T+1)\right]\right\} \\
& +\frac{C\left(1-\mathrm{body}, 1-\mathrm{body}\left[21^{\Omega-2}\right] 0\right)}{2 \Omega(\Omega+1)(\Omega-1)}\left\{n(\Omega+2)\left(\left(\Omega-\frac{1}{2} n\right) \cdots 2 \Omega T(T+1)\right\}\right. \\
& +\frac{2 C\left(\mathrm{aa}, \mathrm{aa}\left[21^{2-2}\right] 0\right)}{3 \Omega(\Omega-1)(\Omega-2)(\Omega+1)}\left\{\frac{9}{8} n(n-2)^{2}(\Omega+2)\left(\Omega-\frac{1}{2} n\right)-(\Omega+2) T^{2}(T+1)^{2}\right. \\
& \left.+T(T+1)\left[2 \Omega^{2}+\frac{7}{2}(n-2) \Omega(\Omega+2)-\frac{1}{2} n(n-2)(11 \Omega+6)\right]\right\} \\
& +\frac{C\left(\mathrm{ss}, \mathrm{ss}\left[21^{\Omega-2}\right] 0\right)}{2 \Omega(\Omega-1)(\Omega+1)}\left\{[n(n+2)-4 T(T+1)]\left[\left(\Omega-\frac{1}{2} n\right) \frac{n+2}{2}+T(T+1)\right]\right\} \\
& -\frac{C\left(\mathrm{aa}, 1-\mathrm{body}\left[21^{\Omega-2}\right] 0\right)}{\Omega(\Omega-1)(\Omega+1)[6(\Omega-2)]^{\frac{1}{2}}}\left\{\frac{3}{2} n(n-2)(\Omega+2)\left(\Omega-\frac{1}{2} n\right)\right. \\
& \left.+2 T(T+1)\left[\Omega^{2}-\Omega\left(\frac{5}{2} n-4\right)-n\right]\right\} \\
& +\frac{C\left(\mathrm{ss}, 1-\mathrm{body}\left[21^{\Omega-2}\right] 0\right)}{\Omega(\Omega-1)(\Omega+1)[2(\Omega+2)]^{\frac{1}{2}}}\left\{(\Omega+2)\left(\Omega-\frac{1}{2} n\right)[n(n+2)-4 T(T+1)]\right\} \\
& -\frac{C\left(\mathrm{ss}, \mathrm{aa}\left[21^{\Omega-2}\right] 0\right)}{\Omega(\Omega-1)(\Omega+1)}\left[\frac{\Omega+2}{3(\Omega-2)}\right]^{\frac{1}{2}}\left\{[n(n+2)-4 T(T+1)]\left[\frac{3}{2}(n-2)\left(\Omega-\frac{1}{2} n\right)-T(T+1)\right]\right\} . \\
&
\end{aligned}
$$

For the single $j$-shell only the first two terms survive. For this case the spectral width has been given previously by French [cf. eq. (7.12) of ref. ${ }^{13}$ )]. In eq. (27) the intensity coefficients, $C\left(\ldots T_{0}=0\right)$, with $\varphi_{0}=$ aa, or $\varphi_{0}=$ one-body can be read from eqs. (21)-(24) if the single-particle parameters $\Delta \varepsilon_{s}$ are replaced by average energies

$$
\Delta \varepsilon_{s} \rightarrow \bar{\varepsilon}_{s}=\frac{1}{2}\left[\left(\varepsilon_{s}\right)_{\text {proton }}+\left(\varepsilon_{s}\right)_{\text {neutron }}\right]
$$


and the proton-proton matrix elements of eq. (20) are replaced by:

$$
W_{J} \rightarrow \sqrt{6}\left(W_{J}^{\prime}=\text { average } T=12 \text {-particle matrix element }\right),
$$

or

$$
V_{\mathrm{pp}} \rightarrow \frac{1}{3} \sqrt{6}\left(V_{\mathrm{pp}}+V_{\mathrm{nn}}+V_{\mathrm{np}}\right)
$$

Intensity coefficients with $\varphi_{0}=$ ss are determined by the $T=0$ two-particle matrix elements:

$$
V_{p q t u J}=\left\langle\left[j_{p} j_{q}\right]_{s} J M_{J} T=0|V|\left[j_{t} j_{u}\right]_{s} J M_{J} T=0\right\rangle,
$$

where $\left[j_{p} j_{q}\right]_{s} J M_{J}$ denotes a normalized angular momentum-coupled state, symmetric in orbital-spin space.

The coefficient $C\left(\mathrm{ss}, \mathrm{ss}\left[21^{\Omega-2}\right] T_{0}=0\right)$ can be read off from the coefficient $C$ (aa, aa $\left.\left[21^{\Omega-2}\right] 1\right)$ of eq. (22), if the $W_{J}$ are replaced by the $V_{J}$, and if the coefficient of eq. (22) is multiplied by an overall factor $2(\Omega-2) /(\Omega+2)$.

The coefficient $C\left(\mathrm{ss}, \mathrm{ss}\left[42^{\Omega-2}\right] T_{0}=0\right)$ can be read off from the coefficient $C\left(\right.$ aa, aa $\left.\left[2^{2} 1^{\Omega-4}\right] 1\right)$ of eq. (24), if the $W_{J}$ are replaced by $V_{3}$, if the coefficient is multiplied by an overall factor of 2 , and if the middle term in eq. (24), involving the double sum $\sum_{J^{\prime}}$, is multiplied by the factor $(\Omega-2) /(\Omega+2)$.

The coefficient $C\left(\mathrm{ss}, 1\right.$-body $\left.\left[21^{\Omega-2}\right] T_{0}=0\right)$ follows from the coefficient $C$ (aa, 1 -body $\left[21^{\Omega-2}\right] 1$ ) of eq. (23), if the replacements $\Delta \varepsilon_{s} \rightarrow \bar{\varepsilon}_{s}$ and $W_{J} \rightarrow V_{J}$ are made, and if the coefficient is multiplied by an overall factor $[2(\Omega-2) /(\Omega+2)]^{\frac{1}{2}}$.

Finally, the coefficient $C\left(\mathrm{ss}, \mathrm{aa}\left[21^{\Omega-2}\right] T_{0}=0\right)$ follows from $C\left(\mathrm{aa}, \mathrm{aa}\left[21^{\Omega-2}\right] 1\right)$ of eq. (22), with replacements such as $W_{\text {rrrrs }} W_{\text {ssss } J^{\prime}} \rightarrow \frac{1}{2}\left[V_{\text {rrrr J }} W_{\text {ssss } y^{\prime}}^{\prime}+W_{\text {rrrrJ }}^{\prime} V_{\text {ssss }}\right]$, if the coefficient of eq. (22) is in addition multiplied by the overall factor $[6(\Omega-2) /$ $(\Omega+2)]^{\frac{2}{2}}$.

\section{4. $[\mathrm{U}(\Omega) \times \mathrm{U}(4)]$ symmetry: An application to the $A=25$ system}

Although results for isospin distributions can be obtained by alternate techniques, a detailed knowledge of the Racah algebra becomes vital if partial widths are to be calculated by simple techniques for the higher unitary groups, $\mathrm{U}(\Omega) \times \mathrm{U}(\mathrm{k})$. The Wigner supermultiplet symmetry $(k=4)$ is of particular interest. The most interesting application will involve the $U(6) \times U(4)$ symmetry of the $2 \mathrm{~s}-1 \mathrm{~d}$ shell since this is an example of a useful symmetry for which considerable symmetry breaking must be expected. To gain a measure of the goodness of space symmetry (or Wigner supermultiplet) quantum numbers in the $2 \mathrm{~s}-1 \mathrm{~d}$ shell, it will be advantageous to calculate the admixture coefficients $\chi^{2}\left([f]\left[f^{\prime}\right]\right)$ of eq. (4) for representations $[f]$ corresponding to the higher spatial symmetries. The partial width formula, eq. (13), can be used to calculate admixture coefficients $\chi^{2}\left([f]\left[f^{\prime}\right]\right)$ for any pair of irreducible representations of $\mathrm{U}(6) \times \mathrm{U}(4)$, provided the Hamiltonian has been decomposed into irreducible tensor components under this symmetry group. An $\mathrm{SU}(3) \times \mathrm{SU}(4)$ tensor decomposition has recently been given ${ }^{8}$ ) for various modifications of the Kuo-Brown interaction 


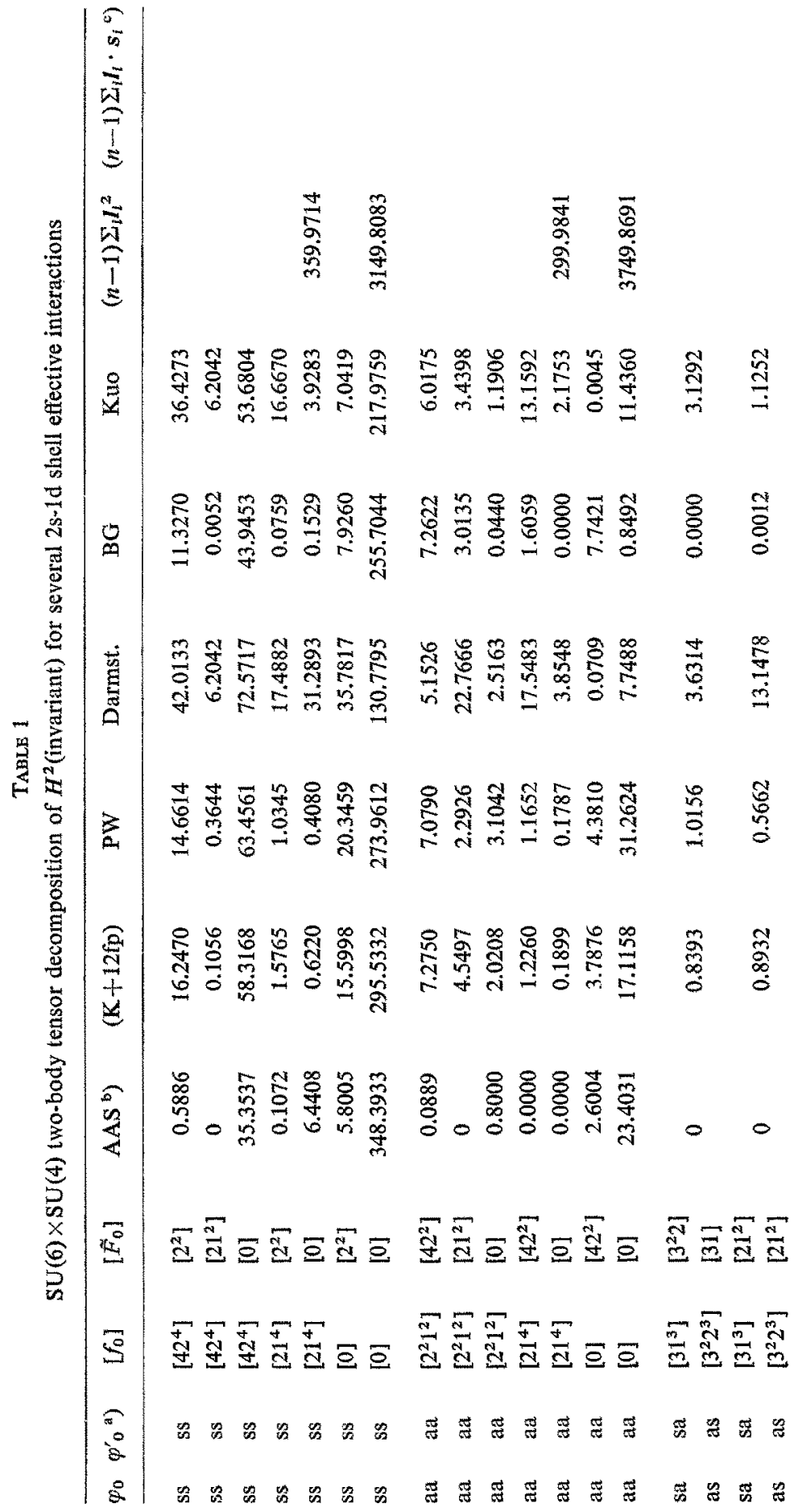




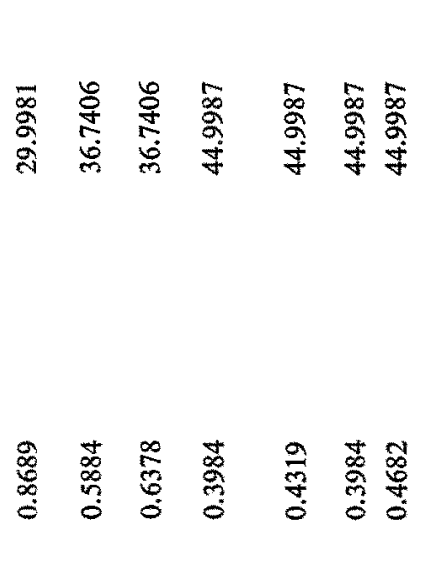

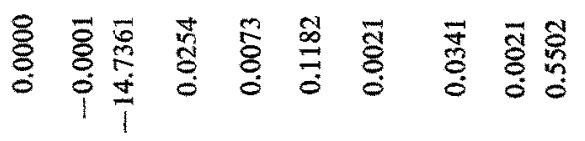

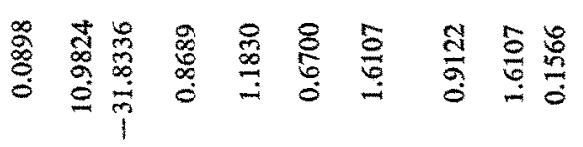

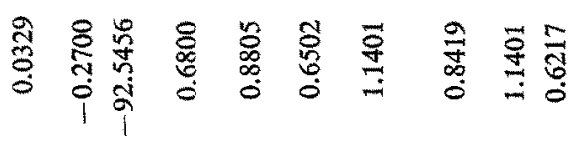

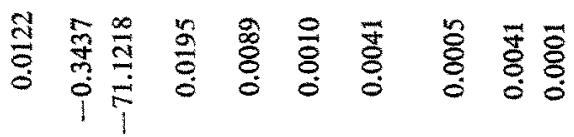

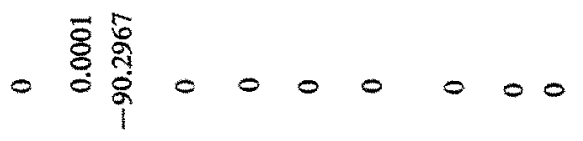

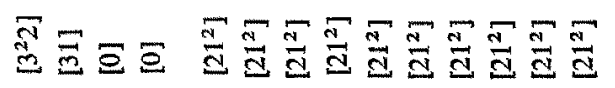

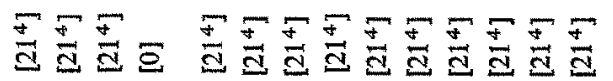

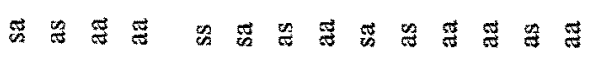

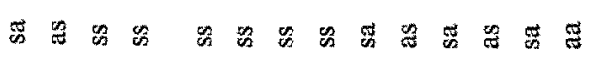

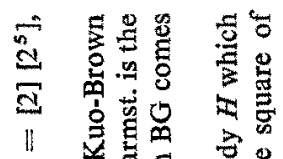

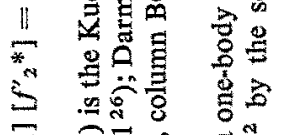

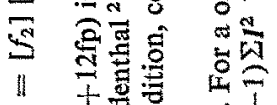

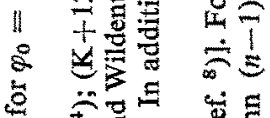

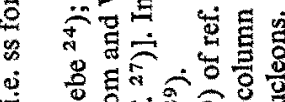

$\stackrel{0}{\circ}$ 영

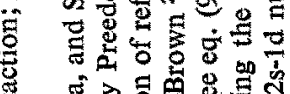

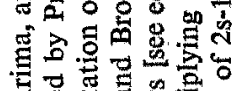

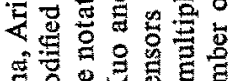

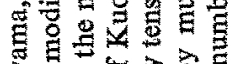

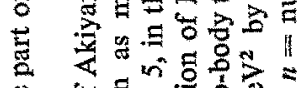

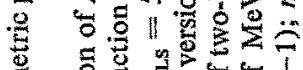

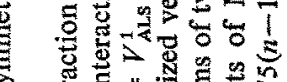

离 氙.

E 当

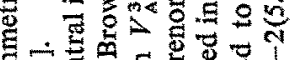

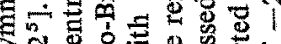

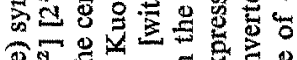

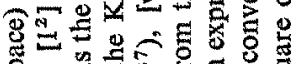

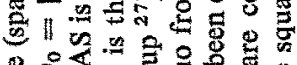

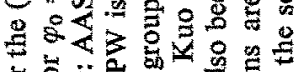

के क्ष

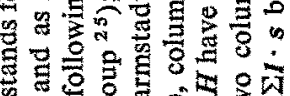

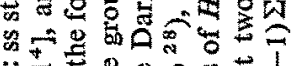

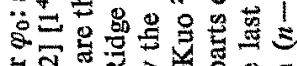

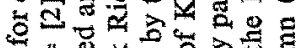

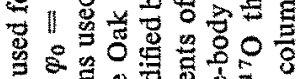

.

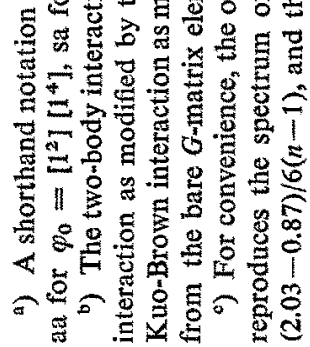


for the $2 \mathrm{~s}-1 \mathrm{~d}$ shell. The full $\mathrm{SU}(6) \times \mathrm{SU}(4)$ tensor decomposition $\left.{ }^{9}\right)$ for these interactions can be achieved by a further coupling of the SU(3) tensor components by means of reduced $\mathrm{SU}(6) \supset \mathrm{SU}(3)$ Wigner coefficients ${ }^{22,23}$ ). The $\mathrm{SU}(6) \times \mathrm{SU}(4)$ content is shown in table 1 for several of the effective two-body interactions which have been used in recent $2 \mathrm{~s}-1 \mathrm{~d}$ shell calculations. The table shows only the $\mathrm{SU}(6) \times$ $\mathrm{SU}(4)$ content of the interactions, the cozfficients $C\left(\varphi_{0} \varphi_{0}^{\prime}\left[f_{0}\right]\left[\widetilde{F}_{0}\right]\right)$ of eq. (9), since this is the quantity of primary interest in the present application. The $\mathrm{SU}(6) \times \mathrm{SU}(4)$ content is obtained by summing over the $\mathrm{SU}(3)$ and $L_{0}$ and $S_{0}$ and $M_{0}$ components of the interactions, [the sum over $\alpha_{0}$ and $\beta_{0}$ of eq. (9)]. For example, the space symmetric-symmetric part of the interaction with $\mathrm{SU}(6) \times \mathrm{SU}(4)$ irreducible tensor components $\left[f_{0}\right]\left[\widetilde{F}_{0}\right]=\left[42^{4}\right]\left[2^{2}\right]$ is obtained by summing over all central $\left[L_{0}=S_{0}\right.$ $=0)$ components of the SU(3) irreducible representations $\left(\lambda_{0} \mu_{0}\right)=(44),(60),(06)$, three independent (22)'s, and (00), as well as over the tensor $\left(L_{0}=S_{0}=2\right)$ components of the representations $\left(\lambda_{0} \mu_{0}\right)=(33),(41),(14),(11)$ besides the (44), (60), $(06),(22)^{3}$, and $(00)$ representations which make up the full $\mathrm{SU}(3)$ content of $\left[f_{0}\right]=$ $\left[42^{4}\right]$. (Note that $\left[\widetilde{F}_{0}\right]=\left[2^{2}\right]$ contains no components with $S_{0} T_{0}=10$. Note also that the interactions of table 1 are all charge independent $\left(T_{0}=0\right.$, no Coulomb contributions). $\mathrm{SU}(6) \times \mathrm{SU}(4)$ tensors of the type [0][21 $\left.{ }^{2}\right]$ are therefore completely missing since the $\mathrm{SU}(6)$ representation [0] contains only $L_{0}=0$ components while the $\mathrm{SU}(4)$ representation $\left[21^{2}\right]$ does not contain $S_{0} T_{0}=00$.)

The two-body interactions which have been singled out are those which have recently been used successfully in extensive shell model calculations in the $2 \mathrm{~s}-1 \mathrm{~d}$ shell. Table 1 includes (i) the central interaction of Akiyama, Arima, and Sebe ${ }^{24}$ ); (ii) the modification of the Kuo-Brown interaction used by the Oak Ridge group ${ }^{25}$ ) in their shell model calculations in the $A-17-24$ region; (iii) a somewhat more drastic modification of the Kuo-Brown interaction obtained by Preedom and Wildenthal ${ }^{25}$ ) from an empirical best fit to nuclei in the $A=18-22$ region; and (iv) a modification of the Kuo-Brown interaction used by the Darmstadt group [Manakos et al. ${ }^{27}$ )] in which the symmetric-antisymmetric and antisymmetric-symmetric parts of the KuoBrown interaction have been increased empirically by rather sizeable factors in order to achieve a fit for the $\mathrm{K}$-band separations in nuclei such as ${ }^{24} \mathrm{Mg}$. Table 1 also gives the SU(6) $\times \mathrm{SU}(4)$ content of the bare $G$-matrix elements of Kuo ${ }^{28}$ ) and the renormalized matrix elements of Kuo and Brown ${ }^{29}$ ). The one-body part of $H$ has been taken from the spectrum of ${ }^{17} \mathrm{O}$. Its $\mathrm{SU}(6) \times \mathrm{SU}(4)$ tensor content can be obtained in one-body form from

$$
\begin{aligned}
& \sum_{i} l_{i} \cdot s_{i}=\sqrt{10} \sum_{M_{0}}(-1)^{1-M_{0}} T_{\left(\lambda_{0} \mu_{0}\right)=(11)\left[L_{0}^{5}=1 M_{0} ; S_{0}=1,-M_{0} T_{0}=0\right.}^{\left[22^{4}\right] ;}, \\
& \sum_{i} l_{i}^{2}=10 \sqrt{6} T_{(00) 00 ;}^{[1]\left[11^{15] ;}[0] ;[0]\right.}-2 \sqrt{30} T_{(22) 00 ;}^{[1][1] 5] ;\left[21^{4}\right] ;[0]}\left[\begin{array}{l}
{[0]} \\
00
\end{array}\right.
\end{aligned}
$$

Alternately, it may be convenient to express the operators $\sum l^{2}, \sum l \cdot s$ in terms of two-body irreducible tensor operators (see table 1). 
Expressions for the centroids have been given by Parikh ${ }^{6}$ ). In terms of the irreducible tensor coefficients of eq. (8) the needed separation between centroids of different $[f]$ (fixed $n$ ) is given by

$$
\begin{aligned}
E_{\mathrm{c}}([f])-E_{\mathrm{c}}\left(\left[f^{\prime}\right]\right)=\left[\frac{1}{2[126]^{\frac{1}{2}}} c(\mathrm{ss}[0][0])-\frac{1}{2[150]^{\frac{1}{2}}} c(\mathrm{aa}[0][0])\right] \\
\times\left[\left\langle G_{2}\right\rangle_{[f]}-\left\langle G_{2}\right\rangle_{\left[f^{\prime}\right]}\right],
\end{aligned}
$$

where the $\mathbf{U}(4)$ Casimir invariant $\left\langle G_{2}\right\rangle_{[f]}$ is given by $\sum_{i} \tilde{f}_{i}^{2}+3 \tilde{f}_{1}+\tilde{f}_{2}-\tilde{f}_{3}-3 \tilde{f}_{4}$. The magnitudes of $\mathrm{c}(\mathrm{ss}[0][0])$ and $\mathrm{c}(\mathrm{aa}[0][0])$ can be read from table 1 by taking the square roots of the corresponding coefficients $C$ (rows 7 and 14 of table 1 , respectively); $c(\mathrm{ss}[0][0])$ is positive, while $c(\mathrm{aa}[0][0])$ is negative for all interactions of table 1 .

The case of nine particles in the $2 \mathrm{~s}-1 \mathrm{~d}$ shell $(A=25)$ has been singled out as a special example. On the one hand the $A=25$ system is possibly beginning to come within reach of modern shell model computer technology without truncation of the $(2 \mathrm{~s}-1 \mathrm{~d})^{n}$ space $\left.^{30,31}\right)$. On the other hand, the spectra of $A=25$ nuclei show well developed rotational bands, and any understanding of the collective nature of the states can come only from a highly limited part of the full shell model space related to the underlying $[S U(6) \supset S U(3)] \times S U(4)$ symmetry. This has recently been demonstrated by a shell model calculation ${ }^{8}$ ) using a truncated basis including only nine favored $S U(3)$ representations from each of the two highest possible spatial symmetries, $[f]=[441]$ and [432]. This calculation has been remarkably successful in reproducing the experimentally observed spectra, [energy spacings, $B(E 2)$ and $B(\mathrm{M} 1)$ values, and the $K_{\mathrm{J}}$ nature of the three lowest positive parity rotational bands]. A vital factor in the success of this calculation, however, has been the introduction of a $(\lambda \mu)$ dependent renormalization of the two-body interaction of the type proposed by Harvey ${ }^{32}$ ) to account for core polarization effects (in place of the $3 p-1 h$ KuoBrown renormalization terms). To gain a further understanding of such renormalization terms, it may therefore also be interesting to study the $A=25$ system with a larger part of the full shell model space and interactions which have been successful in lighter nuclei using the full $(2 \mathrm{~s}-1 \mathrm{~d})^{n}$ spacc. Since calculations in an $[\mathrm{SU}(6) \supset \mathrm{SU}(3)]$ $\times \mathrm{SU}(4)$ basis with a much larger part of the shell model space are still prohibitively time consuming, it will be advantageous to use spectral averaging techniques to give at least a qualitative or semiquantitative measure of the admixtures of lower space symmetries $\left[f^{\prime}\right]$ into the higher space symmetries, particularly $[f]=[441]$ which is the predominant component for the low energy part of the spectrum. For this purpose the admixture coefficients $\chi^{2}\left([f],\left[f^{\prime}\right]\right)$ have been calculated for the four highest spatial symmetries $[f]=[441]$, [432], [4311], and [333], and all possible $\left[f^{\prime}\right]$. Results are shown in table 2 for the interactions (1)-(4) of table 1 . Although the admixture coeffcients can give reliable quantitative information only when $\chi^{2} \ll 1$, in which case $\chi^{2}([f]$, $\left.\left[f^{\prime}\right]\right)$ measures the total intensity of the admixture of all states of $\left[f^{\prime}\right]$ into an average state of $[f]$, the results of table 2 can be used as a qualitative guide to indicate how the full shell model space can be truncated in terms of representations $[f]$ of SU(6). 


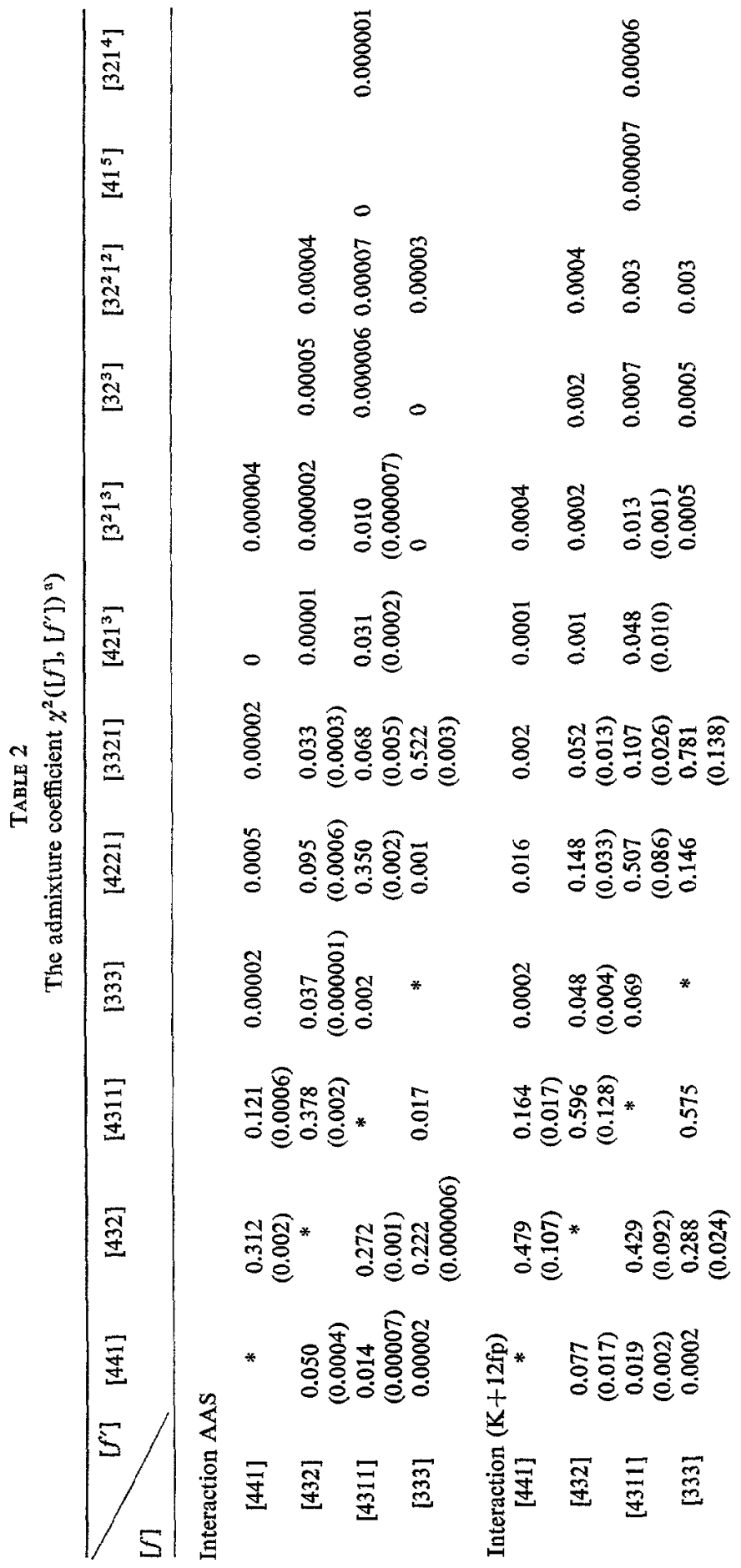


喜 홍

$\tilde{8}$
0
$\delta$
$\delta$
$\delta$

$\frac{0}{8} \delta$

兽 $\stackrel{n}{0} \stackrel{⿱}{0}$

룽

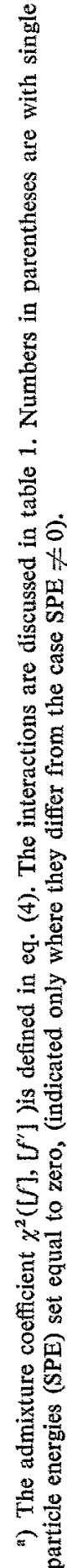

䓀

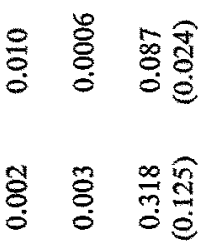

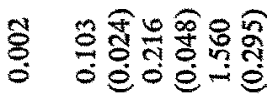

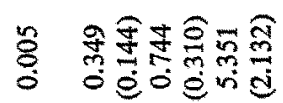

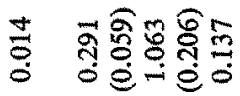

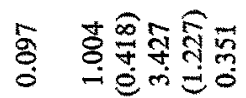

양 융융

용 웡웡

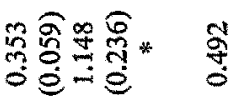

पूลู̊ป

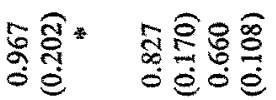

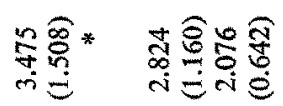

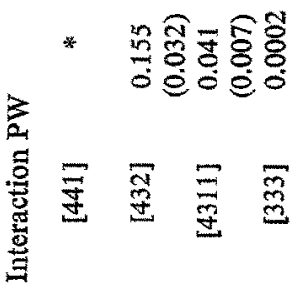

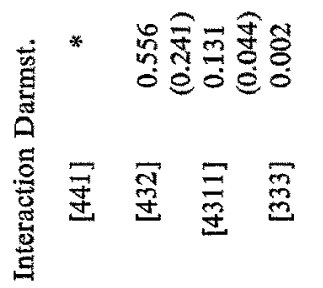


Table 2 shows that the two-body part of the central interaction used by AAS leads to remarkably little mixing of different space symmetries [ $f]$. For this interaction the mixing arises mainly from the single-particle part of $H$ (the spin orbit term), leading to strong admixture coefficients for only one or two $\left[f^{\prime}\right]$ for each $[f]$. For the $(K+12 f p)$ interaction the two-body part of the interaclion makes important contributions to the symmetry breaking which reinforce the effect of the one-body spin orbit term. However, admixture coefficients are important only for symmetries $[f]$ and $\left[f^{\prime}\right]$ which can be connected by tensors of $\left[21^{4}\right]$ symmetry, and each $[f]$ has strong connections to only one or two $\left[f^{\prime}\right]$. The symmetry breaking is even stronger for the PW interaction and becomes so large for the Darmstadt interaction that the SU(6) $\times \mathrm{SU}(4)$ symmetry must be expected to break down completely for this interaction, precluding a truncation of the shell model space in terms of the space symmetries $[f]$. For the $(\mathrm{K}+12 \mathrm{fp})$ or AAS interactions, however, a truncation in terms of quantum numbers $[f]$ should be expected to be valid. The only symmetries with significant direct admixtures into the dominant highest symmetry $[f]=[441]$ are the symmetries $\left[f^{\prime}\right]=[432]$ and possibly [4311]. Since [432] is itself strongly connected to [4311] it should be expected that a valid truncation of the shell model space would have to include at least the three space symmetries $[f]=[441],[432]$, and [4311]. Since [4311] again has strong connections to [4221], and this symmetry has strong connections to even lower symmetries, even a truncation in terms of the symmetries [441], [432], and [4311] may be open to question. Here it becomes important to bear in mind that the coefficients of table 2 give a measure of the admixture of $\left[f^{\prime}\right]$ to an average state of $[f]$. Since a strong subgroup symmetry, $\mathrm{SU}(3)$, plays an important role, the low energy states of [441] are far from average states but are predominantly states of high $\mathrm{SU}(3)$ symmetry (large values of $\lambda$ and $\mu$ ), in particular $(\lambda \mu)=(66)$ and (93). States of symmetry $\left[f^{\prime}\right]$ can therefore be expected to lead to significant admixtures for the lowest states of [441] symmetry only if $\left[f^{\prime}\right]$ is connected to [441], directly or indirectly, by large admixture coefficients, and only if $\left[f^{\prime}\right]$ contains states of high SU(3) symmetry $(\lambda \mu)$. The only such states for the space symmetry [4221] come from the single $\mathrm{SU}(3)$ representation $(\lambda \mu)=(82)$; and $\mathrm{SU}(6)$ representations of even lower space symmetry contain no states of very high $\mathrm{SU}(3)$ symmetry. The detailed shell model calculations of ref. ${ }^{8}$ ) show that admixtures of $(\lambda \mu)=(82)$ are quite unimportant for the three lowest rotational bands, dominated by $(\lambda \mu)=(66)$ and $(93)$, so that a truncation scheme based on the space symmetries [441], [432], and [4311] should be valid. Since both [432] and [4311] contain an $\mathrm{SU}(3)$ representation $(\lambda \mu)=(74)$ which has strong connections to (93) and (66) through the simplest SU(3) symmetry breaking terms of the interaction, it must, however, be expected that some states from both [4311] and [432] symmetries must be included in the shell model space for the $A=25$ system.

Although the partial width formula presented in this investigation does lead to a very simple $a$ priori test for the goodness of $\mathrm{SU}(\Omega) \times \mathrm{SU}(4)$ symmetry for a major nuclear shell, the example of the $A=25$ system does point up the additional compli- 
cations which arise if there is a strong symmetry associated with a subgroup of a higher symmetry group. Unfortunately it has not yet been possible to derive a formula for admixture coefficients for the representations of a subgroup imbedded in the $\mathrm{SU}(\Omega) \times \mathrm{SU}(4)$ group of a simplicity comparable to that derived in this investigation for the admixture coefficients for the $\mathrm{SU}(\Omega) \times \mathrm{SU}(4)$ group itself. Further work remains to be done to develop a simple, reliable, and complete a priori measure for the admixture of group representations.

It is a pleasure to acknowledge conversations with J. B. French and J. C. Parikh which formed the stimulus for much of this work.

\section{Appendix 1}

\section{DERIVATION OF THE PARTIAL WIDTH FORMULA}

The $n$-particle state vectors are labeled by the irreducible representation labels of $\mathrm{U}(\Omega) \times \mathrm{U}(k),|[f] \alpha \beta\rangle$, where $\alpha$ stands for a complete set of subgroup labels for $\mathrm{U}(\Omega), \beta$ for a complete set of subgroup labels for $\mathrm{U}(k)$. Operators are built from single-particle creation and annihilation operators with $[\mathrm{U}(\Omega) \times \mathrm{U}(k)]$ irreducible tensor character $a_{\alpha \beta}^{+}=t_{\alpha \beta}^{[1][1]}, a_{\alpha \beta}=(-1)^{\eta(\alpha)+\eta(\beta)} t_{\alpha^{*} \beta^{*}}^{\left.[1 *] 11^{*}\right]}$, with $\left[1^{*}\right]=\left[1^{\Omega-1}\right]$ for $\mathrm{U}(\Omega)$ and $\left[1^{*}\right]=\left[1^{k-1}\right]$ for $\mathrm{U}(k)$. The phase factors $\eta(\alpha)$ and $\eta(\beta)$ are as always somewhat dependent on phase conventions. Since the final result for the partial width formula is independent of these phase factors, no specific phase conventions need be specified. Two-particle operators are coupled to components with definite irreducible tensor character by

$$
\left[\boldsymbol{a}^{+} \times \boldsymbol{a}^{+}\right]_{\alpha_{2} \beta_{2}}^{\left[f_{2}\right]\left[\tilde{f}_{2}\right]}=\sum_{\alpha_{1} \gamma^{\prime} 1} \sum_{\beta_{1} \beta^{\prime} 1}\left\langle[1] \alpha_{1}[1] \alpha_{1}^{\prime} \mid\left[f_{2}\right] \alpha_{2}\right\rangle\left\langle[1] \beta_{1}[1] \beta_{1}^{\prime} \mid\left[\tilde{f}_{2}\right] \beta_{2}\right\rangle a_{\alpha_{1} \beta_{1}}^{+} a_{\alpha^{\prime} 1 \beta^{\prime} 1}^{+},
$$

where the coefficients are full Wigner coefficients for unitary groups $\mathrm{U}(\Omega)$ and $\mathrm{U}(k)$, respectively; and an analogous expression is used to construct the coupled pair an-

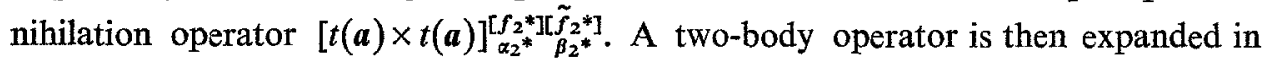
terms of the basic $\mathrm{U}(\Omega) \times \mathrm{U}(k)$ irreducible tensor operators

$$
\begin{aligned}
& T_{z_{0} \beta_{0}}^{\varphi_{0}\left[f_{0}\right]\left[\tilde{F}_{0}\right]}=\sum_{\alpha_{2} \alpha^{\prime} 2^{*} \beta_{2} \beta_{2}^{\prime} z^{*}}\left\langle\left[f_{2}^{\prime *}\right] \alpha_{2}^{\prime *}\left[f_{2}\right] \alpha_{2} \mid\left[f_{0}\right] \alpha_{0}\right\rangle \\
& \times\left\langle\left[\tilde{f}_{2}^{\prime *}\right] \beta_{2}^{\prime *}\left[\tilde{f}_{2}\right] \beta_{2} \mid\left[\tilde{F}_{0}\right] \beta_{0}\right\rangle\left[\boldsymbol{a}^{+} \times \boldsymbol{a}^{+}\right]_{\alpha_{2} \beta_{2}}^{\left[f_{2}\right]\left[\tilde{f}_{2}\right]}[t(\boldsymbol{a}) \times t(\boldsymbol{a})]_{\alpha^{\prime} 2^{*} \beta^{\prime} 2^{*}}^{\left[f^{\prime} * \tilde{f}^{\prime} z^{*}\right]} \text {. }
\end{aligned}
$$

where $\varphi_{0}$ is a shorthand notation for $\left[f_{2}\right]\left[f_{2}^{\prime *}\right]$, see fig. 1. (Note that the Wigner coefficients are free of multiplicity labels if $\left[f_{2}\right]\left(\left[\tilde{f}_{2}\right]\right)$ are restricted to two- or oneparticle representations.) The Hamiltonian can be expanded in terms of the basic irreducible tensor operators $T^{\varphi_{0}\left[f_{0}\right]\left[\tilde{F}_{0]}\right]}$, [see eq. (8) of the text]. In terms of the irreducible tensor components of $H$, the operator $H^{2}$ can be written

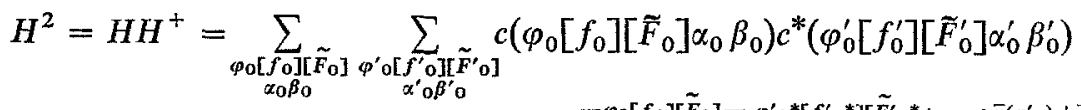

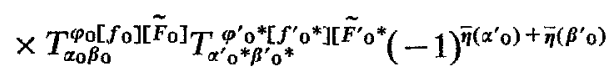


(It is possible but not necessary to choose the phase factors $\bar{\eta}=\eta$. Since final results are again independent of these phase factors, no specific choices need to be made.) The product of two $\mathrm{U}(\Omega) \times \mathrm{U}(k)$ irreducible tensors can be coupled further to resultant irreducible tensor character $\left[f_{00}\right]\left[\widetilde{F}_{00}\right]$ of the product tensor by means of $U(\Omega)$ and $\mathrm{U}(k)$ Wigner coefficients. Since the average of $H^{2}$ is determined solely by the $U(\Omega)$ and $\mathrm{U}(k)$ invariant parts of this operator, only invariant coupled tensor operators are needed

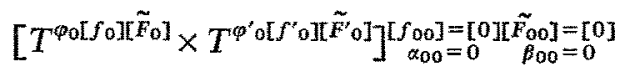

$$
\begin{aligned}
& =\sum_{\alpha_{0} \alpha^{\prime} 0} \sum_{\beta_{0} \beta^{\prime}{ }^{\prime}}\left\langle\left[f_{0}\right] \alpha_{0}\left[f_{0}^{\prime}\right] \alpha_{0}^{\prime} \mid[0] 0\right\rangle\left\langle\left[\widetilde{F}_{0}\right] \beta_{0}\left[\tilde{F}_{0}^{\prime}\right] \beta_{0}^{\prime} \mid[0] 0\right\rangle
\end{aligned}
$$

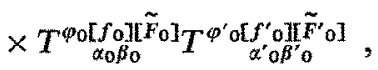

where

$$
\begin{aligned}
& \left\langle\left[f_{0}\right] \alpha_{0}\left[f_{0}^{\prime}\right] \alpha_{0}^{\prime} \mid[0] 0\right\rangle=\delta_{\left[f^{\prime} 0 H f^{*_{0} 0}\right]} \delta_{\alpha^{\prime}{ }^{\prime} \alpha^{*_{0}}}(-1)^{\eta\left(\alpha_{0}\right)}\left[\operatorname{dim}\left[f_{0}\right]\right]^{-\frac{1}{2}},
\end{aligned}
$$

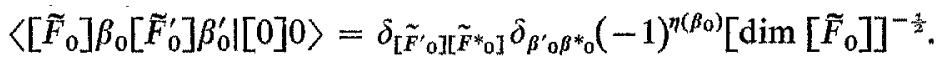

The invariant part of $H^{2}$ can then be written

$$
\begin{aligned}
& H^{2}(\text { invariant })=\sum_{\varphi_{0} \varphi^{\prime} 0} \sum_{\left[f_{0}\right]\left[\tilde{F}_{0}\right]} \frac{C\left(\varphi_{0} \varphi_{0}^{\prime}\left[f_{0}\right]\left[\tilde{F}_{0}\right]\right)}{\operatorname{dim}\left[f_{0}\right] \operatorname{dim}\left[\tilde{F}_{0}\right]}
\end{aligned}
$$

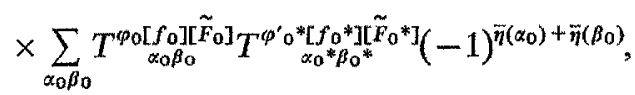

where the intensity coefficient, $C\left(\varphi_{0} \varphi_{0}^{\prime}\left[f_{0}\right]\left[\widetilde{F}_{0}\right]\right)$, is defined by eq. (9) of the text, and where eq. (A.4) and its inverse have been used, along with the reality of the $c$ coefficients.

The decomposition into the partial widths, defined in eq. (3), is then accomplished by expressing the average of $H^{2}$ in terms of

$$
\begin{aligned}
& \sum_{\alpha \beta}\left\langle[f] \alpha \beta\left|H^{2}\right|[f] \alpha \beta\right\rangle=\sum_{\left[\tilde{f}^{\prime}\right]} \sum_{\varphi_{0} \varphi^{\prime}\left[\tilde{f}_{0}\right]\left[\tilde{F}_{0}\right]} \frac{C\left(\varphi_{0} \varphi_{0}^{\prime}\left[f_{0}\right]\left[\tilde{F}_{0}\right]\right)}{\operatorname{dim}\left[f_{0}\right] \operatorname{dim}\left[\tilde{F}_{0}\right]} \\
& \times \sum_{\substack{\alpha \beta \alpha \beta \\
\alpha^{\prime} \beta^{\prime}}}\left\langle[f] \alpha \beta\left|T^{\varphi_{0}\left[f_{0}\right]\left[\tilde{F}_{0} \beta_{0}\right]}\right|\left[f^{\prime}\right] \alpha^{\prime} \beta^{\prime}\right\rangle\left\langle[f] \alpha \beta\left|T^{\varphi^{\prime} 0\left[f_{0}\right]\left[\tilde{F}_{0}\right]}\right|\left[f^{\prime}\right] \alpha^{\prime} \beta^{\prime}\right\rangle,
\end{aligned}
$$

where we have used

$$
\begin{aligned}
\left\langle\left[f^{\prime}\right] \alpha^{\prime} \beta^{\prime}\left|T^{\varphi^{\prime} 0_{0}^{*}\left[f_{0}^{*}\right]\left[\tilde{F}_{0}{ }^{*} \beta_{0}^{*}\right]}(-1)^{\bar{\eta}\left(\alpha_{0}\right)+\bar{\eta}\left(\beta_{0}\right)}\right|[f] \alpha \beta\right\rangle & \\
& =\langle[f] \alpha \beta| T_{\alpha_{0} \varphi_{0}^{\prime}\left[f_{0}\right]\left[\tilde{F_{0}}\right]}\left[\left[f^{\prime}\right] \alpha^{\prime} \beta^{\prime}\right\rangle^{*},
\end{aligned}
$$

and the reality of the matrix elements of $T^{\left.\varphi_{0}\left[f_{0}\right] I \tilde{F}_{0}\right]}$.

The sums over subgroup labels $\alpha, \beta$ are easily evaluated if the matrix elements of $\boldsymbol{T}$ are expressed in terms of unitary group Wigner and Racah coefficients. The matrix 
element between two $n$-particle states $|[f] \alpha \beta\rangle$ is reduced to the matrix elements of two-particle states by the usual c.f.p. expansion, where the full $n$ to $n-2$ particle c.f.p. is factored into two parts, one for the $\mathrm{U}(\Omega)$ symmetry, the second for the $\mathrm{U}(k)$ symmetry. With $k=4$, the factoring of the full c.f.p. is the familiar one into a space and a spin-isospin part, and is given by ${ }^{33,34}$ )

$$
\left.\left.\left.\left.\left[\frac{\mathscr{N}_{\left[f^{\prime \prime}\right]}}{\mathscr{N}_{[f]}}\right]^{\frac{1}{2}}\left\langle\left[f^{\prime \prime}\right] \bar{\alpha}^{\prime \prime} L^{\prime \prime}\left[f_{2}\right] \bar{\alpha}_{2} L_{2}\right|\right\}[f] \bar{\alpha} L\right\rangle \times\left\langle\left[\tilde{f}^{\prime \prime}\right] \bar{\beta}^{\prime \prime} S^{\prime \prime} T^{\prime \prime}\left[\tilde{f}_{2}\right] S_{2} T_{2}\right|\right\}[\tilde{f}] \bar{\beta} S T\right\rangle
$$

Here $\mathscr{N}_{[f]}$ is the dimension of the irreducible representation $[f]$ of the permutation group of $n$ particles, $\mathscr{N}_{\left[f^{\prime \prime}\right]}$ the dimension of $\left[f^{\prime \prime}\right]$ for the permutation group of $n-2$ particles ${ }^{\dagger}$. The labels $\bar{\alpha}$, have been introduced for the moment: $\bar{\alpha}$ denotes all $\mathrm{U}(\Omega)$ subgroup labels other than $L M_{L}$; similarly for $\bar{\beta}$. If the space part of the c.f.p. is combined with the angular momentum vector coupling coefficient, the resultant coefficient can be identified as a unitary group Wigner coefficient for $\mathrm{U}(\Omega)$,

$$
\left.\left.\left\langle\left[f^{\prime \prime}\right] \bar{\alpha}^{\prime \prime} L^{\prime \prime}\left[f_{2}\right] \bar{\alpha}_{2} L_{2}\right|\right\}[f] \bar{\alpha} L\right\rangle\left\langle L^{\prime \prime} M_{L}^{\prime \prime} L_{2} M_{L_{2}} \mid L M_{L}\right\rangle=\left\langle\left[f^{\prime \prime}\right] \alpha^{\prime \prime}\left[f_{2}\right] \alpha_{2} \mid[f] \alpha\right\rangle,
$$

similarly for the spin-isospin part of the c.f.p. In terms of such unitary group Wigner coefficients the c.f.p. expansion for the matrix element of a two-body tensor operator is then given by

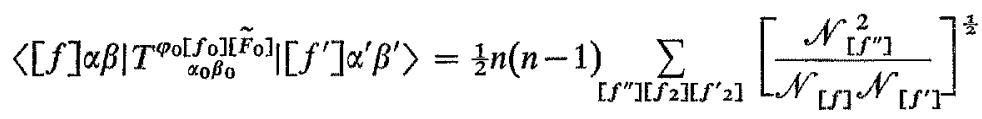

$$
\begin{aligned}
& \times \sum_{\substack{\alpha^{\prime \prime} \beta^{\prime \prime} \\
\alpha_{2} \beta_{2} \alpha_{2}^{\prime} \beta^{\prime} \beta_{2}}}\left\langle\left[f^{\prime \prime}\right] \alpha^{\prime \prime}\left[f_{2}\right] \alpha_{2} \mid[f] \alpha\right\rangle\left\langle\left[f^{\prime \prime}\right] \alpha^{\prime \prime}\left[f_{2}^{\prime}\right] \alpha_{2}^{\prime} \mid\left[f^{\prime}\right] \alpha^{\prime}\right\rangle \\
& \times\left\langle\left[\tilde{f}^{\prime \prime}\right] \beta^{\prime \prime}\left[\tilde{f}_{2}\right] \beta_{2} \mid[\tilde{f}] \beta\right\rangle\left\langle\left[\tilde{f}^{\prime \prime}\right] \beta^{\prime \prime}\left[\tilde{f}_{2}^{\prime}\right] \beta_{2}^{\prime} \mid\left[\tilde{f}^{\prime}\right] \beta^{\prime}\right\rangle \\
& \times\left\langle\left[f_{2}\right] \alpha_{2} \beta_{2}\left|T_{\alpha_{0} \beta_{0}}^{\varphi_{0}\left[f_{0}^{0}\right]\left[\tilde{F_{0}}\right.}\right|\left[f_{2}^{\prime}\right] \alpha_{2}^{\prime} \beta_{2}^{\prime}\right\rangle \text {. }
\end{aligned}
$$

The two-particle matrix elements of $T$ can be evaluated from the defining eq. (A.2) to give

$$
\begin{aligned}
\left.\left.\left\langle\left[f_{2}\right] \alpha_{2} \beta_{2}\right| T_{\alpha_{0} \beta_{0}}^{\varphi_{0}\left[f_{0}\right]\left[\tilde{F}_{0}\right]}\right]\left[f_{2}^{\prime}\right] \alpha_{2}^{\prime} \beta_{2}^{\prime}\right\rangle=-2(-1)^{\eta\left(\alpha_{2}^{\prime}\right)+\eta\left(\beta^{\prime}\right)} \\
\\
\quad \times \delta_{\varphi_{0},\left[f_{2}\right]\left[f^{\prime}{ }_{2}\right]}\left\langle\left[f_{2}^{\prime *}\right] \alpha_{2}^{\prime *}\left[f_{2}\right] \alpha_{2} \mid\left[f_{0}\right] \alpha_{0}\right\rangle\left\langle\left[\tilde{f}_{2}^{\prime *}\right] \beta_{2}^{\prime *}\left[\tilde{f}_{2}\right] \beta_{2} \mid\left[\tilde{F}_{0}\right] \beta_{0}\right\rangle .
\end{aligned}
$$

† It should perhaps be pointed out that this factoring into space and spin-isospin parts, based on the phase conventions of Jahn and Van Wieringen ${ }^{33}$ ) or Elliott, Hope and $\mathrm{Jahn}^{34}$ ), assumes that the states of $[f]$ and $[f]$ transform contragrediently under a permutation of the $n$ particles; see, e.g., eq. (1C-23) of Bohr and Mottelson ${ }^{35}$ ). It is often more convenient to use space and spin-isospin functions which transform identically under a permutation of the $n$ particles. With this convention the above $n$ to $n-2$ particle c.f.p. must be multiplied by an additional phase factor $(-1)^{*}$; see, e.g. a footnote in ref. ${ }^{8}$ ). This phase factor is important in determining the phase of a specific matrix element. However, since $\chi$ is a function only of $\left[f^{\prime \prime}\right],\left[f_{2}\right]$, and $[f]$, it can be adsorbed into the phase conventions of the unitary group Wigner coefficients. Since the present applications, which depend only on averages of products of matrix elements, will be shown to be independent of such phase conventions, all results will be independent of $\psi$. 
To evaluate the sums over subgroup labels $\alpha^{\prime \prime}, \beta^{\prime \prime} \alpha_{2} \ldots$ it is convenient to make use of a symmetry property of the unitary group Wigner coefficients

$$
\begin{aligned}
& \left\langle\left[f^{\prime \prime}\right] \alpha^{\prime \prime}\left[f_{2}^{\prime}\right] \alpha_{2}^{\prime} \mid\left[f^{\prime}\right] \alpha^{\prime}\right\rangle \\
& \quad=\left[\frac{\operatorname{dim}\left[f^{\prime}\right]}{\operatorname{dim}\left[f^{\prime \prime}\right]}\right]^{\frac{1}{2}}(-1)^{\eta\left(\alpha_{2}^{\prime}\right)+\varphi\left(\left[f^{\prime \prime} 3\left[f^{\prime} 2\right]\left[f^{\prime}\right]\right)\right.}\left\langle\left[f^{\prime}\right] \alpha^{\prime}\left[f_{2}^{\prime *}\right] \alpha_{2}^{\prime *} \mid\left[f^{\prime \prime}\right] \alpha^{\prime \prime}\right\rangle,
\end{aligned}
$$

and the analagous relation for the $\mathrm{U}(k)$ Wigner coefficients. As in all such symmctry relations, the phase factor must include the conjugation phase factor $\eta\left(\alpha_{2}^{\prime}\right)$ but is subject to phase conventions also in its overall dependence on $\left[f^{\prime \prime}\right],\left[f_{2}^{\prime}\right]$, and $\left[f^{\prime}\right]$. With eqs. (A.10) and (A.11), the sums over subgroup labels of eq. (A.9) can be expressed in terms of products of three $\mathrm{U}(\Omega)$ Wigner coefficients and products of three $\mathrm{U}(k)$ Wigner coefficients. Such sums can be evaluated most economically by a generalization to the higher unitary groups of the well known result for angular momentum vector coupling coefficients:

$$
\begin{aligned}
\sum_{M_{2} M_{3} M_{12}} & \left\langle L_{1} M_{1} L_{2} M_{2} \mid L_{12} M_{12}\right\rangle\left\langle L_{12} M_{12} L_{3} M_{3} \mid L M\right\rangle \\
& \times\left\langle L_{2} M_{2} L_{3} M_{3} \mid L_{23} M_{23}\right\rangle \\
= & \left\langle L_{1} M_{1} L_{23} M_{23} \mid L M\right\rangle U\left(L_{1} L_{2} L L_{3} ; L_{12} L_{23}\right) .
\end{aligned}
$$

The corresponding sum for the Wigner coefficients of the unitary group $U(\Omega)$ is

$$
\begin{aligned}
\sum_{\alpha_{2} \alpha_{2}^{\prime} z^{\prime \prime}}\left\langle\left[f^{\prime}\right] \alpha^{\prime}\left[f_{2}^{\prime *}\right] \alpha_{2}^{\prime *} \mid\left[f^{\prime \prime}\right] \alpha^{\prime \prime}\right\rangle\left\langle\left[f^{\prime \prime}\right] \alpha^{\prime \prime}\left[f_{2}\right] \alpha_{2} \mid[f] \alpha\right\rangle \\
\\
\quad \times\left\langle\left[f_{2}^{\prime *}\right] \alpha_{2}^{\prime *}\left[f_{2}\right] \alpha_{2} \mid\left[f_{0}\right] \alpha_{0}\right\rangle \\
=\sum_{\rho}\left\langle\left[f^{\prime}\right] \alpha^{\prime}\left[f_{0}\right] \alpha_{0} \mid[f] \alpha\right\rangle_{\rho} U\left(\left[f^{\prime}\right]\left[f_{2}^{* *}\right][f]\left[f_{2}\right] ;\left[f^{\prime \prime}\right]\left[f_{0}\right] \rho\right) .
\end{aligned}
$$

Here, the $U$-coefficient is a unitary group Racah coefficient ${ }^{15-18}$ ) written in a notation which is a straightforward generalization of that for the ordinary angular momentum recoupling coefficient in unitary form. Since the direct product $\left[f^{\prime}\right]$ $\times\left[f_{0}\right]$ is in general not simply reducible, both the unitary group Wigner and Racah coefficients will depend on a multiplicity label $\rho$. The $\alpha$-sum cannot be expressed as a product of a single Wigner and Racah coefficient but involves a sum over the multiplicity label $\rho$. With the use of (A.13) and the corresponding result for the $U(k)$ coefficients, the matrix element of a tensor operator $T^{\varphi_{0}\left[\tilde{f}_{0}\right]\left[\tilde{F}_{0}\right]}$ can be put into compact form. The result still depends on phase factors such as $\varphi\left(\left[f^{\prime \prime}\right]\left[f_{2}^{\prime}\right]\left[f^{\prime}\right]\right)$ and therefore seems to be complicated by phase conventions. Such phase factors, however, can be eliminated by expressing the final result in terms of ratios of Racah coefficients. Setting $\left[f_{0}\right]=[0]$ in eq. (A.13), we note that

$$
\begin{aligned}
U\left(\left[f^{\prime}\right]\left[f_{2}^{\prime *}\right]\left[f^{\prime}\right]\left[f_{2}^{\prime}\right] ;\left[f^{\prime \prime}\right][0]\right) & =(-1)^{\varphi\left(\left[f^{\prime \prime}\right]\left[f^{\prime} 2\right]\left[f^{\prime}\right]\right)}\left[\frac{\operatorname{dim}\left[f^{\prime \prime}\right]}{\operatorname{dim}\left[f^{\prime}\right] \operatorname{dim}\left[f_{2}^{\prime}\right]}\right]^{\frac{1}{2}} .
\end{aligned}
$$


The matrix element of a $\mathrm{U}(\Omega) \times \mathrm{U}(4)$ irreducible two-body tensor can then be expressed in the following compact form

$$
\begin{aligned}
& \langle[f] \times \beta| T_{\alpha_{0} \beta_{0}}^{\varphi_{0}\left[f_{0}\right]\left[\tilde{F}_{0}\right]}\left[\left[f^{\prime}\right] \alpha^{\prime} \beta^{\prime}\right\rangle=\mathscr{F}(n) \sum_{\left[\tilde{f}^{\prime \prime}\right]}\left[\frac{\mathscr{N}_{\left[f^{\prime \prime}\right]}^{2}}{\tilde{N}_{[f]} \mathscr{N}_{\left[f^{\prime}\right]}}\right]^{\frac{1}{2}} \\
& \times\left[\frac{1}{\operatorname{dim}\left[\tilde{f}_{2}^{\prime}\right] \operatorname{dim}\left[\tilde{f}_{2}^{\prime}\right]}\right]^{\frac{1}{2}} \sum_{\rho} \frac{U\left(\left[f^{\prime}\right]\left[f_{2}^{\prime *}\right][f]\left[f_{2}\right] ;\left[f^{\prime \prime}\right]\left[f_{0}\right] \rho\right)}{U\left(\left[\tilde{f}^{\prime}\right]\left[f_{2}^{\prime *}\right]\left[f^{\prime}\right]\left[f_{2}^{\prime}\right] ;\left[f^{\prime \prime}\right][0]\right)}\left\langle\left[f^{\prime}\right] \alpha^{\prime}\left[f_{0}\right] \alpha_{0} \mid[f] \alpha\right\rangle_{\rho} \\
& \times \sum_{\tilde{\rho}} \frac{U\left(\left[\tilde{f}^{\prime}\right]\left[\tilde{f}_{2}^{\prime *}\right][\tilde{f}]\left[\tilde{f}_{2}\right] ;\left[\tilde{f}^{\prime \prime}\right]\left[\tilde{F}_{0}\right] \tilde{\rho}\right)}{U\left(\left[\tilde{f}^{\prime}\right]\left[\tilde{f}_{2}^{\prime *}\right]\left[\tilde{f}^{\prime}\right]\left[\tilde{f}_{2}^{\prime}\right] ;\left[\tilde{f}^{\prime \prime}\right][0]\right)}\left\langle\left[\tilde{f}^{\prime}\right] \beta^{\prime}\left[\tilde{F}_{0}\right] \beta_{0} \mid[\tilde{f}] \beta\right\rangle_{\beta}
\end{aligned}
$$

with $\mathscr{F}(n)=-n(n-1)$. A similar expression holds for the matrix element of a onebody operator $\left(\varphi_{0} \equiv[1]\left[1^{\Omega-1}\right]\right)$, if the factor $-n(n-1)$ is replaced by $\mathscr{F}(n)=+n$, and if $\left[f^{\prime \prime}\right]$ are interpreted as $(n-1)$ particle representations, while $\left[f_{2}\right]$ must in this case denote the representations [1]. By expressing the final matrix element in terms of the above ratio of Racah coefficients, the result becomes independent of specific phase conventions chosen for the unitary group Wigner coefficients.

Although the sum over multiplicity labels complicates the evaluation of any one specific matrix element, it will actually lead to simplicity when such matrix elements are averaged over an irreducible representation [f]. Using (A.15), the sum over subgroup labels in (A.7) becomes

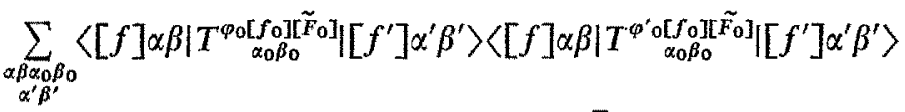

$$
\begin{aligned}
& =\mathscr{F}(n) \mathscr{F}^{\prime}(n) \sum_{\left.\left[f^{\prime \prime}{ }_{2}\right] f^{\prime \prime}{ }_{1}\right]} \frac{\mathscr{N}_{\left[f^{\prime \prime}{ }^{\prime}\right]} \mathscr{N}_{\left[f^{\prime \prime}\right]}}{\mathscr{N}_{\left[f^{\prime}\right]} \mathscr{N}_{\left[f^{\prime}\right]}^{\prime}}\left[\frac{1}{\operatorname{dim}\left[f_{2}^{\prime}\right] \operatorname{dim}\left[\tilde{f}_{2}^{\prime}\right] \operatorname{dim}\left[f_{1}^{\prime}\right] \operatorname{dim}\left[\tilde{f}_{1}^{\prime}\right]}\right]^{\frac{1}{2}} \\
& \times \sum_{\rho \rho^{\prime}} \frac{U\left(\left[f^{\prime}\right]\left[f_{2}^{\prime *}\right][f]\left[f_{2}\right] ;\left[f_{2}^{\prime \prime}\right]\left[f_{0}\right] \rho\right)}{U\left(\left[f^{\prime}\right]\left[f_{2}^{\prime *}\right]\left[f^{\prime}\right]\left[f_{2}^{\prime}\right] ;\left[f_{2}^{\prime \prime}\right][0]\right)} \frac{U\left(\left[f^{\prime}\right]\left[f_{1}^{\prime *}\right][f]\left[f_{1}\right] ;\left[f_{1}^{\prime \prime}\right]\left[f_{0}\right] \rho^{\prime}\right)}{U\left(\left[f^{\prime}\right]\left[f_{1}^{\prime *}\right]\left[f^{\prime}\right]\left[f_{1}^{\prime}\right] ;\left[f_{1}^{\prime \prime}\right][0]\right)} \\
& \times \sum_{\alpha^{\prime} \alpha 0 \alpha}\left\langle\left[f^{\prime}\right] \alpha^{\prime}\left[f_{0}\right] \alpha_{0} \mid[f] \alpha\right\rangle_{p}\left\langle\left[f^{\prime}\right] \alpha^{\prime}\left[f_{0}\right] \alpha_{0} \mid[f] \alpha\right\rangle_{\rho^{\prime}} \\
& \times \sum_{\tilde{\rho} \tilde{\rho}^{\prime}} \frac{U\left(\left[\tilde{f}^{\prime}\right]\left[\tilde{f}_{2}^{\prime *}\right][\tilde{f}]\left[\tilde{f}_{2}\right] ;\left[\tilde{f}_{2}^{\prime}\right]\left[\tilde{F}_{0}\right] \tilde{\rho}\right)}{U\left(\left[\tilde{f}^{\prime}\right]\left[\tilde{f}_{2}^{\prime *}\right]\left[\tilde{f}^{\prime}\right]\left[\tilde{f}_{2}^{\prime}\right] ;\left[\tilde{f}_{2}^{\prime \prime}\right][0]\right)} \frac{U\left(\left[\tilde{f}^{\prime}\right]\left[\tilde{f}_{1}^{\prime *}\right][\tilde{f}]\left[\tilde{f}_{1}\right] ;\left[\tilde{f}_{1}^{\prime \prime}\right]\left[\tilde{F}_{0}\right] \tilde{\rho}^{\prime}\right)}{U\left(\left[\tilde{f}^{\prime}\right]\left[\tilde{f}_{1}^{\prime *}\right]\left[\tilde{f}^{\prime}\right]\left[\tilde{f}_{1}^{\prime}\right] ;\left[\tilde{f}_{1}^{\prime \prime}\right][0]\right)} \\
& \times \sum_{\beta^{\prime} \beta_{0} \beta}\left\langle\left[\tilde{f}^{\prime}\right] \beta^{\prime}\left[\tilde{F}_{0}\right] \beta_{0} \mid[\tilde{f}] \beta\right\rangle_{\tilde{\rho}}\left\langle\left[\tilde{f}^{\prime}\right] \beta^{\prime}\left[\tilde{F}_{0}\right] \beta_{0} \mid[\tilde{f}] \beta\right\rangle_{\tilde{\rho}^{\prime}} .
\end{aligned}
$$

The sums over subgroup labels can now be carried out, using the orthonormality of the unitary group Wigner coefficients

$$
\begin{aligned}
& \sum_{\alpha}\left(\sum_{\alpha^{\prime} z_{0}}\left\langle\left[f^{\prime}\right] \alpha^{\prime}\left[f_{0}\right] \alpha_{0} \mid[f] \alpha\right\rangle_{\rho}\left\langle\left[f^{\prime}\right] \alpha^{\prime}\left[f_{0}\right] \alpha_{0} \mid[f] \alpha\right\rangle_{\rho^{\prime}}\right)=\delta_{\rho \rho^{\prime}} \operatorname{dim}[f], \\
& \sum_{\beta}\left(\sum_{\tilde{\beta}^{\prime} \beta_{0}}\left\langle\left[\tilde{f}^{\prime}\right] \beta^{\prime}\left[\tilde{F}_{0}\right] \beta_{0} \mid[\tilde{f}] \beta\right\rangle_{\tilde{\rho}}\left\langle\left[\tilde{f}^{\prime}\right] \beta^{\prime}\left[\tilde{F}_{0}\right] \beta_{0} \mid[\tilde{f}] \beta\right\rangle_{\tilde{\rho}^{\prime}}\right)=\delta_{\tilde{\rho} \tilde{\rho}^{\prime}} \operatorname{dim}[\tilde{f}] .
\end{aligned}
$$

This finally leads to the partial width formula, eq. (13) of the text. 


\section{Appendix 2}

TABULATION OF RACAH COEFFICIENT SUMS

Although expressions for the Racah coefficients of the unitary groups $\mathrm{U}(N)$ with $N>2$ are complicated by the multiplicity structure ${ }^{15-18}$ ), the only quantities needed in the present application are the sums $\Sigma\left(\left[f^{\prime}\right]\left[f_{0}\right][f] ;\left[f_{2}^{\prime *}\right]\left[f_{2}\right]\left[f_{2}^{\prime \prime}\right]\right.$; $\left.\left[f_{1}^{\prime *}\right]\left[f_{1}\right]\left[f_{1}^{\prime \prime}\right]\right)$ of eq. (13b) involving the products of Racah coefficients of $\mathrm{U}(N)$ summed over the multiplicity label $\rho$. Such sums are independent of the details of the multiplicity structure. They are functions only of $N$ and the axial distances, $\tau_{a b}$, where

$$
\tau_{a b}=f_{a}-f_{b}-a+b .
$$

Compact expressions for the sums $\Sigma$ have recently been derived by permutation group techniques ${ }^{19}$ ). The derivations and more general tabulations will be given elsewhere $\left.{ }^{19}\right)$. In the present application the only nontrivial case with $\left[f^{\prime}\right] \neq[f]$ involves the representations

$$
\left[f^{\prime}\right]=\left[f\left(a^{3} b c^{2} d^{2} e^{2} \ldots\right)\right]=\left[f\left(a^{2} c d e . . .\right)\right],
$$

where the notation implies that the tableau for $\left[f^{\prime}\right]$ is obtained from the tableau for $[f]$ by first adding two squares to every row of $[f]$, then removing three squares from row $a$, one square from row $b$, two squares from rows $c, d, e$, and all remaining rows. Similarly, $\left[f^{\prime \prime}\right]=[f(a b)]$ is the tableau obtained by removing one square from rows $a$ and $b$ of the tableau for $[f]$. The quantities $\Sigma$ are tabulated as functions of $N$ and $\tau_{a b}$ in table 3 for the special case $\left[f^{\prime}\right]=\left[f\left(a^{3} b c^{2} d^{2} e^{2} \ldots\right)\right]$. The only representation $\left[f_{0}\right]$ for which it is possible to have $\left[f_{1}^{\prime}\right] \neq\left[f_{2}^{\prime}\right],\left[f_{1}\right] \neq\left[f_{2}\right]$ is the representation $\left[f_{0}\right]=\left[21^{N-2}\right]$. Even for this case only the sums $\Sigma$ with $\left[f_{1}^{\prime}\right]=\left[f_{2}^{\prime}\right],\left[f_{1}\right]=\left[f_{2}\right]$ are tabulated, since the remaining possibilities can (in the special case $\left[f^{\prime}\right]=$ $\left.\left[f\left(a^{3} b c^{2} d^{2} e^{2} \ldots\right)\right]\right)$ be obtained from

$$
\begin{aligned}
\Sigma\left(\left[f^{\prime}\right]\left[21^{N-2}\right][f] ;\left[f_{2}^{\prime *}\right]\left[f_{2}\right]\left[f_{2}^{\prime \prime}\right] ;\left[1^{N-1}\right][1]\left[f_{1}^{\prime \prime}\right]\right) \\
=(-1)^{\prime}\left[\Sigma\left(\left[f^{\prime}\right]\left[21^{N-2}\right][f] ;\left[f_{2}^{\prime *}\right]\left[f_{2}\right]\left[f_{2}^{\prime \prime}\right] ;\left[f_{2}^{\prime *}\right]\left[f_{2}\right]\left[f_{2}^{\prime \prime}\right]\right)\right. \\
\left.\quad \times \Sigma\left(\left[f^{\prime}\right]\left[21^{N-2}\right][f] ;\left[1^{N-1}\right][1]\left[f_{1}^{\prime \prime}\right] ;\left[1^{N-1}\right][1]\left[f_{1}^{\prime \prime}\right]\right)\right]^{\frac{1}{2}}
\end{aligned}
$$

with $v=0$ for $\left[f_{2}^{\prime}\right]=\left[1^{2}\right]$, and $v=1$ for $\left[f_{2}^{\prime}\right]=[2]$, and

$$
\begin{aligned}
& \Sigma\left(\left[f^{\prime}\right]\left[21^{N-2}\right][f] ;\left[f_{2}^{\prime *}\right]\left[f_{2}\right]\left[f_{2}^{\prime \prime}\right] ;\left[f_{1}^{\prime *}\right]\left[f_{1}\right]\left[f_{1}^{\prime \prime}\right]\right) \\
&=(-1)^{\prime}\left[\Sigma\left(\left[f^{\prime}\right]\left[21^{N-2}\right][f] ;\left[f_{2}^{\prime *}\right]\left[f_{2}\right]\left[f_{2}^{\prime \prime}\right] ;\left[f_{2}^{\prime *}\right]\left[f_{2}\right]\left[f_{2}^{\prime \prime}\right]\right)\right. \\
&\left.\times \Sigma\left(\left[f^{\prime}\right]\left[21^{N-2}\right][f] ;\left[f_{1}^{\prime *}\right]\left[f_{1}\right]\left[f_{1}^{\prime \prime}\right] ;\left[f_{1}^{\prime *}\right]\left[f_{1}\right]\left[f_{1}^{\prime \prime}\right]\right)\right]^{\frac{1}{2}}
\end{aligned}
$$

with $v=0$ for $\left[f_{2}^{\prime}\right]\left[f_{1}^{\prime}\right]=[2][2]$ or $\left[1^{2}\right]\left[1^{2}\right]$, and $v=1$ for $\left[f_{2}^{\prime}\right]\left[f_{1}^{\prime}\right]=[2]\left[1^{2}\right]$ or $\left[1^{2}\right][2]$. [Note, however, that these simple relations hold only for the special case $\left[f^{\prime}\right]=\left[f\left(a^{3} b c^{2} d^{2} e^{2} \ldots\right)\right]$. For the case $\left[f^{\prime}\right]=[f]$, for example, the sums with $\left[f_{1}^{\prime}\right] \neq\left[f_{2}^{\prime}\right],\left[f_{1}\right] \neq\left[f_{2}\right]$ are in general more complicated $\left.{ }^{19}\right)$.] 
TABLE 3

The sums $\Sigma\left(\left[f^{\prime}\right]\left[f_{0}\right][f] ;\left[f_{2}^{\prime *}\right]\left[f_{2}\right]\left[f^{\prime \prime}\right] ;\left[f_{2}^{\prime *}\right]\left[f_{2}\right]\left[f^{\prime \prime}\right]\right)$ with $\left[f^{\prime}\right]=$

$$
\left.=\left[f\left(a^{3} b c^{2} d^{2} e^{2} \ldots\right)\right]^{\mathrm{a}}\right)
$$

$\left[f^{\prime \prime}\right]\left[\tilde{f}^{\prime \prime}\right] \quad \Sigma$ with $\left[f_{0}\right]=\left[21^{N-2}\right],\left[f_{2}^{\prime *}\right]\left[f_{2}\right]=\left[1^{N-1}\right][1]$

$[f(a)][f(a)] \quad N \prod_{b}^{\prime} \frac{\tau_{a b-2}}{\tau_{a b}-1}$

$\left[f^{\prime \prime}\right]\left[\tilde{f}^{\prime \prime}\right] \quad \Sigma$ with $\left[f_{0}\right]=\left[21^{N-2}\right],\left[f_{2}^{\prime *}\right]\left[f_{2}\right]=\left[2^{N-1}\right][2]$

$[f(a a)][f(a a)] \quad \frac{N(N+1)}{N+2} \prod_{b}^{\prime} \frac{\tau_{a b}-3}{\tau_{a b}-1}$

$[f(a b)][f(a b)] \quad \frac{N(N+1)}{N+2} \prod_{b}^{\prime} \frac{\left(\tau_{a b}-2\right)\left(\tau_{a b}+1\right)}{\tau_{a b}\left(\tau_{a b}-1\right)}$

$[f(a c)][f(a c)] \quad \frac{N(N+1)}{2(N+2)} \prod_{b}^{\prime} \frac{\left(\tau_{a b}-2\right)\left(\tau_{a c}+1\right)\left(\tau_{b c}+2\right)}{\left(\tau_{a b}-1\right) \tau_{a c}\left(\tau_{b c}+1\right)}$

$[f(a a)][f(a b)] \quad \frac{N(N+1)}{N+2} \prod_{b}^{\prime}\left[\frac{\left(\tau_{a b}-3\right)\left(\tau_{a b}-2\right)\left(\tau_{a b}+1\right)}{\left(\tau_{a b}-1\right)^{2} \tau_{a b}}\right]^{\frac{1}{2}}$

$[f(a a)][f(a c)] \quad \frac{N(N+1)}{N+2} \prod_{b}^{\prime}\left[\frac{\left(\tau_{a b}-2\right)\left(\tau_{a b}-3\right)\left(\tau_{a c}+1\right)\left(\tau_{b c}+2\right)}{2\left(\tau_{a b}-1\right)\left(\tau_{a b}-1\right) \tau_{a c}\left(\tau_{b c}+1\right)}\right]^{\frac{1}{2}}$

$[f(a b)][f(a c)] \quad \frac{N(N+1)}{N+2} \prod_{b}^{\prime} \frac{\left(\tau_{a b}-2\right)}{\left(\tau_{a b}-1\right)}\left[\frac{\left(\tau_{a b}+1\right)\left(\tau_{a c}+1\right)\left(\tau_{b c}+2\right)}{2 \tau_{a b} \tau_{a c}\left(\tau_{b c}+1\right)}\right]^{\frac{1}{2}}$

$[f(a d)][f(a c)] \frac{N(N+1)}{2(N+2)} \Pi_{b}^{\prime} \frac{\left(\tau_{a b}-2\right)}{\left(\tau_{a b}-1\right)}\left[\frac{\left(\tau_{a c}+1\right)\left(\tau_{a d}+1\right)\left(\tau_{b c}+2\right)\left(\tau_{b d}+2\right)}{\tau_{a c} \tau_{a d}\left(\tau_{b c}+1\right)\left(\tau_{b d}+1\right)}\right]^{\frac{1}{2}}$

$\left[f^{\prime \prime}\right]\left[\bar{f}^{\prime \prime}\right] \quad \Sigma$ with $\left[f_{0}\right]=\left[21^{N-2}\right],\left[f_{2}^{\prime *}\right]\left[f_{2}\right]=\left[1^{N-2}\right]\left[1^{2}\right]$

$[f(a c)][f(a c)] \quad \frac{N(N-1)}{2(N-2)} \Pi_{b}^{\prime} \frac{\left(\tau_{a b}-2\right)\left(\tau_{a c}-1\right) \tau_{b c}}{\left(\tau_{a b}-1\right) \tau_{a c}\left(\tau_{b c}+1\right)}$

$[f(a d)][f(a c)] \quad \frac{N(N-1)}{2(N-2)} \prod_{b}^{\prime} \frac{\tau_{a b}-2}{\tau_{a b}-1}\left[\frac{\left(\tau_{a c}-1\right)\left(\tau_{a d}-1\right) \tau_{b c} \tau_{b d}}{\tau_{a c} \tau_{a d}\left(\tau_{b c}+1\right)\left(\tau_{b d}+1\right)}\right]^{\frac{1}{2}}$ 
$\left[f^{\prime \prime}\right]\left[\tilde{f}^{\prime \prime}\right] \quad \Sigma$ with $\left[f_{0}\right]=\left[21^{N-2}\right],\left[f_{2}^{\prime *}\right]\left[f_{2}\right]=\left[2^{N-1}\right]\left[1^{2}\right]$

$[f(a b)][f(a b)] \quad(N+1) \prod_{b} \frac{\tau_{a b}-2}{\tau_{a b}}$

$[f(a b)][f(a c)] \quad(N+1) \prod_{b}\left[\frac{\left(\tau_{a b}-2\right)^{2}\left(\tau_{a c}-1\right)\left(\tau_{b c}+2\right)}{2 \tau_{a b}\left(\tau_{a b}-1\right) \tau_{a c}\left(\tau_{b c}+1\right)}\right]^{\frac{1}{2}}$

$[f(a c)][f(a c)] \quad \frac{N+1}{2} \prod_{b}^{\prime} \frac{\left(\tau_{a b}-2\right)\left(\tau_{a c}-1\right)\left(\tau_{b c}+2\right)}{\left(\tau_{a b}-1\right) \tau_{a c}\left(\tau_{b c}+1\right)}$

$[f(a d)][f(a c)] \quad \frac{N+1}{2} \prod_{b}^{\prime} \frac{\left(\tau_{a b}-2\right)}{\left(\tau_{a b}-1\right)}\left[\frac{\left(\tau_{a c}-1\right)\left(\tau_{a d}-1\right)\left(\tau_{b c}+2\right)\left(\tau_{b d}+2\right)}{\tau_{a c} \tau_{a d}\left(\tau_{b c}+1\right)\left(\tau_{b d}+1\right)}\right]^{\frac{3}{2}}$

$\left[f^{\prime \prime}\right]\left[\bar{f}^{\prime \prime}\right] \quad \Sigma$ with $\left[f_{0}\right]=\left[21^{N-2}\right],\left[f_{2}^{\prime *}\right]\left[f_{2}\right]=\left[1^{N-2}\right][2]$

$[f(a c)][f(a c)] \quad \frac{N-1}{2} \prod_{b}^{\prime} \frac{\left(\tau_{a b}-2\right)\left(\tau_{a c}+1\right) \tau_{b c}}{\left(\tau_{a b}-1\right) \tau_{a c}\left(\tau_{b c}+1\right)}$

$[f(a a)][f(a c)] \quad N-1 \prod_{b}^{\prime}\left[\frac{\left(\tau_{a b}-2\right)\left(\tau_{a c}+1\right) \tau_{b c}}{2\left(\tau_{a b}-1\right) \tau_{a c}\left(\tau_{b c}+1\right)}\right]^{\frac{1}{2}}$

$[f(a a)][f(a a)] \quad(N-1) \prod_{b}^{\prime}$

$[f(a d)][f(a c)] \quad \frac{N-1}{2} \prod_{b}^{\prime} \frac{\tau_{a b}-2}{\tau_{a b}-1}\left[\frac{\left(\tau_{a c}+1\right)\left(\tau_{a d}+1\right) \tau_{b c} \tau_{b d}}{\tau_{a c} \tau_{a d}\left(\tau_{b c}+1\right)\left(\tau_{b d}+1\right)}\right]^{\frac{1}{2}}$

$\left[f^{\prime \prime}\right]\left[f^{\prime \prime}\right] \quad \Sigma$ with $\left[f_{0}\right]=\left[42^{N-2}\right],\left[f_{2}^{\prime *}\right]\left[f_{2}\right]=\left[2^{N-1}\right][2]$

$[f(a a)][f(a a)] \quad N(N+1) \prod_{b} \prod^{\prime}\left\{\frac{1}{2} \prod_{\substack{i \neq a \\ i \neq b}} \frac{\left(\tau_{a i}-1\right)}{\left(\tau_{a i}-2\right)}-\frac{1}{N+2} \frac{\tau_{a b}-3}{\tau_{a b}-1}\right\}$

$[f(a b)][f(a b)] \quad N(N+1) \prod_{b}^{\prime} \frac{\tau_{a b}-2}{\tau_{a b}}\left\{\frac{1}{2} \prod_{\substack{i \neq a \\ i \neq b}} \frac{\tau_{b i}}{\left(\tau_{b i}-1\right)}-\frac{1}{N+2} \frac{\tau_{a b}+1}{\tau_{a b}-1}\right\}$

$[f(a c)][f(a c)] \quad \frac{N(N+1)}{2} \prod_{b}^{\prime} \frac{\tau_{a b}-2}{\tau_{a b}-1}\left\{\frac{\left(\tau_{a c}-1\right) \tau_{b c}}{\tau_{a c}\left(\tau_{b c}+1\right.} \prod_{\substack{i \neq a \\ i \neq b \\ i \neq c}} \frac{\tau_{c i}}{\left(\tau_{c i}-1\right)}-\frac{1}{N+2} \frac{\left(\tau_{a c}+1\right)\left(\tau_{b c}+2\right)}{\tau_{a c}\left(\tau_{b c}+1\right)}\right\}$ 
TABle 3 (continued)

$$
\begin{aligned}
& {[f(a a)][f(a b)]-\frac{N(N+1)}{N+2} \prod_{b}^{\prime}\left[\frac{\left(\tau_{a b}-3\right)\left(\tau_{a b}-2\right)\left(\tau_{a b}+1\right)}{\left(\tau_{a b}-1\right)^{2} \tau_{a b}}\right]^{\frac{1}{2}}} \\
& {[f(a a)][f(a c)]-\frac{N(N+1)}{N+2} \prod_{b}^{\prime}\left[\frac{\left(\tau_{a b}-2\right)\left(\tau_{a b}-3\right)\left(\tau_{a c}+1\right)\left(\tau_{b c}+2\right)}{2\left(\tau_{a b}-1\right)^{2} \tau_{a c}\left(\tau_{b c}+1\right)}\right]^{\frac{1}{2}}} \\
& {[f(a b)][f(a c)]-\frac{N(N+1)}{N+2} \prod_{b}^{\prime} \frac{\tau_{a b}-2}{\tau_{a b}-1}\left[\frac{\left(\tau_{a b}+1\right)\left(\tau_{a c}+1\right)\left(\tau_{b c}+2\right)}{2 \tau_{a b} \tau_{a c}\left(\tau_{b c}+1\right)}\right]^{\frac{1}{2}}} \\
& {[f(a d)][f(a c)]-\frac{N(N+1)}{2(N+2)} \prod_{b}^{\prime} \frac{\tau_{a b}-2}{\tau_{a b}-1}\left[\frac{\left(\tau_{a c}+1\right)\left(\tau_{a d}+1\right)\left(\tau_{b c}+2\right)\left(\tau_{b d}+2\right)}{\tau_{a c} \tau_{a d}\left(\tau_{b c}+1\right)\left(\tau_{b d}+1\right)}\right]^{\frac{1}{2}}}
\end{aligned}
$$

$\left[f^{\prime \prime}\right]\left[f^{\prime \prime}\right] \quad \Sigma$ with $\left[f_{0}\right]=\left[2^{2} 1^{N-4}\right],\left[f_{2}^{\prime *}\right]\left[f_{2}\right]=\left[1^{N-2}\right]\left[1^{2}\right]$

$[f(a c)][f(a c)] \quad \frac{N(N-1)}{2} \prod_{b}^{\prime} \frac{\left(\tau_{a b}-2\right)\left(\tau_{a c}-1\right) \tau_{b c}}{\left(\tau_{a b}-1\right) \tau_{a c}\left(\tau_{b c}+1\right)}\left(\prod_{\substack{i \neq a \\ i \neq b \\ i \neq c}} \frac{\tau_{c i}}{\left(\tau_{c i}-1\right)}-\frac{1}{N-2}\right)$

$[f(a d)][f(a c)]-\frac{N(N-1)}{2(N-2)} \prod_{b}^{\prime} \frac{\tau_{a b}-2}{\tau_{a b}-1}\left[\frac{\left(\tau_{a c}-1\right)\left(\tau_{a d}-1\right) \tau_{b c} \tau_{b d}}{\tau_{a c} \tau_{a d}\left(\tau_{b c}+1\right)\left(\tau_{b d}+1\right)}\right]^{\frac{1}{2}}$

$\left[f^{\prime \prime}\right]\left[\bar{f}^{\prime \prime}\right] \quad \Sigma$ with $\left[f_{0}\right]=\left[3^{2} 2^{N-3}\right],\left[f_{2}^{\prime *}\right]\left[f_{2}\right]=\left[2^{N-1}\right]\left[1^{2}\right]$

$[f(a b)][f(a b)] \quad \frac{N(N+1)}{2} \frac{\tau_{a b}-2}{\tau_{a b}} \prod_{b}^{\prime}\left\{\prod_{\substack{i \neq a \\ i \neq b}} \frac{\tau_{b i}}{\left(\tau_{b i}-1\right)}-\frac{2}{N}\right\}$

$[f(a b)][f(a c)]-(N+1) \prod_{b}^{\prime}\left[\frac{\left(\tau_{a b}-2\right)^{2}\left(\tau_{b c}+2\right)\left(\tau_{a c}-1\right)}{2 \tau_{a b}\left(\tau_{a b}-1\right)\left(\tau_{b c}+1\right) \tau_{a c}}\right]^{\frac{1}{2}}$

$[f(a c)][f(a c)] \quad \frac{N(N+1)}{2} \frac{\left(\tau_{a b}-2\right)\left(\tau_{a c}-1\right) \tau_{b c}}{\left(\tau_{a b}-1\right) \tau_{a c}\left(\tau_{b c}+1\right)} \prod_{b}^{\prime}\left\{\prod_{\substack{i \neq a \\ i \neq b \\ i \neq c}} \frac{\tau_{c i}}{\left(\tau_{c i}-1\right)}-\frac{1}{N} \frac{\tau_{b c}+2}{\tau_{b c}}\right\}$

$[f(a d)][f(a c)]-\frac{N+1}{2} \prod_{b}^{\prime} \frac{\tau_{a b}-2}{\tau_{a b}-1}\left[\frac{\left(\tau_{a c}-1\right)\left(\tau_{a d}-1\right)\left(\tau_{b c}+2\right)\left(\tau_{b d}+2\right)}{\tau_{a c} \tau_{a d}\left(\tau_{b c}+1\right)\left(\tau_{b d}+1\right)}\right]^{\frac{1}{2}}$ 
$\left[f^{\prime \prime}\right]\left[f^{\prime \prime}\right] \quad \Sigma$ with $\left[f_{0}\right]=\left[31^{N-3}\right],\left[f_{2}^{\prime *}\right]\left[f_{2}\right]=\left[1^{N-2}\right][2]$

$[f(a c)][f(a c)] \quad \frac{N(N-1)}{2} \frac{\left(\tau_{a b}-2\right)\left(\tau_{a c}-1\right) \tau_{b c}}{\left(\tau_{a b}-1\right) \tau_{a c}\left(\tau_{b c}+1\right)} \prod_{b}^{\prime}\left\{\prod_{\substack{i \neq a \\ i \neq b \\ i \neq c}} \frac{\tau_{c i}}{\left(\tau_{c i}-1\right)}-\frac{1}{N} \frac{\tau_{a c}+1}{\tau_{a c}-1}\right\}$

$[f(a a)][f(a c)] \quad-(N-1) \prod_{b}^{\prime}\left[\frac{\left(\tau_{a b}-2\right)\left(\tau_{a c}+1\right) \tau_{b c}}{2\left(\tau_{a b}-1\right) \tau_{a c}\left(\tau_{b c}+1\right)}\right]^{\frac{1}{2}}$

$[f(a a)][f(a a)] \quad \frac{N(N-1)}{2} \prod_{b}^{\prime}\left\{\prod_{\substack{i \neq a \\ i \neq b}} \frac{\left(\tau_{a i}-1\right)}{\left(\tau_{a i}-2\right)}-\frac{2}{N}\right\}$

$[f(a d)][f(a c)]-\frac{N-1}{2} \prod_{b}^{\prime} \frac{\tau_{a b}-2}{\tau_{a b}-1}\left[\frac{\left(\tau_{a c}+1\right)\left(\tau_{a d}+1\right) \tau_{b c} \tau_{b d}}{\tau_{a c} \tau_{a d}\left(\tau_{b c}+1\right)\left(\tau_{b d}+1\right)}\right]^{\frac{1}{2}}$

$\left.{ }^{a}\right)(\ldots)=\sum_{\rho} \frac{U\left(\left[f^{\prime}\right]\left[f_{2}^{\prime *}\right][f]\left[f_{2}\right] ;\left[f^{\prime \prime}\right] ;\left[f_{0}\right] \rho\right)}{U\left(\left[f^{\prime}\right]\left[f_{2}^{\prime *}\right]\left[f^{\prime}\right]\left[f_{2}^{\prime}\right] ;\left[f^{\prime \prime}\right] ;[0]\right)} \frac{U\left(\left[f^{\prime}\right]\left[f_{2}^{\prime *}\right][f]\left[f_{2}\right] ;\left[\bar{f}^{\prime \prime}\right] ;\left[f_{0}\right] \rho\right)}{U\left(\left[f^{\prime}\right]\left[f_{2}^{\prime *}\right]\left[f^{\prime}\right]\left[f_{2}^{\prime}\right] ;\left[\bar{f}^{\prime \prime}\right] ;[0]\right)}$

In table 3 the following shorthand notation has been used:

$$
\prod_{b}^{\prime}=\prod_{\substack{i \neq a \\ i \neq b}}\left(1+1 / \tau_{b i}\right)
$$

that is, the product runs over the $N-2$ indices $i$ other than $i=a$ and $b$.

\section{References}

1) J. B. French, in Nuclear structure, ed. A. Hossain, Harunar-Raschid and M. Islam (NorthHolland, Amsterdam, 1967)

2) J. B. French and K. F. Ratcliff, Phys. Rev: C3 (1971) 94, 117

3) F. S. Chang, J. B. French and T. H. Thio, Ann. of Phys. 66 (1971) 137

4) J. C. Parikh, Lectures on group symmetries in nuclear structure, Univ. of Rochester report UR-875-350 (1971)

5) J. B. French and J. C. Parikh, Phys. Lett. 35B (1971) 1

6) J. C. Parikh, Ann. of Phys. 76 (1973) 202

7) J. C. Parikh; Phys. Lett. 41B (1972) 468

8) J. P. Draayer, Nucl. Phys. A216 (1973) 457

9) Z. Pluhar, Nucl. Phys. A167 (1971) 33

10) O. Bohigas, C. Quesne and R. Arvieu, Phys. Lett. 26B (1968) 562

11) K. T. Hecht, Ann. Rev. Nucl. Sci. 23 (1973)

12) J. C. Parikh and S. S. M. Wong, Nucl.'Phys. Al8z (1972) 593

13) J. B. French, in Isospin in nulear physics, ed. D. H. Wilkinson (North-Holland, Amsterdam 1969) 
14) B. G. Wybourne, Symmetry principles and atomic spectroscopy (Wiley Interscience, 1970)

15) J. D. Louck and L. C. Biedenharn, J. Math. Phys. 11 (1970) 2368, 14 (1973) 1336 and earlier references quoted therein

16) K. T. Hecht, Nucl. Phys. 62 (1965) 1

17) K. T. Hecht and S. C. Pang, J. Math. Phys. 10 (1969) 1571

18) J. A. Castilho-Alcaras, L. C. Biedenharn, K. T. Hecht and G. Neely, Ann. of Phys. 60 (1970) 85

19) K. T. Hecht, to be published.

20) J. Jänecke, in Isospin in nuclear physics, ed. D. H. Wilkinson (North-Holland, Amsterdam, 1969)

21) J. M. Soper, in Isospin in Nuclear. Physics, ed. D. H. Wilkinson (North-Holland, Amsterdam, 1969)

22) Y. Akiyama, Nucl. Data A2 (1967) 403

23) J. D. Vergados, dissertation, University of Michigan (1968), Technical Report 07591-2-T

24) Y. Akiyama, A. Arima and T. Sebe, Nucl. Phys. A138 (1969) 273

25) E. C. Halbert, J. B. McGrory, B. H. Wildenthal and S. P. Pandya, Advances in nuclear physics, vol. 4, ed. M. Baranger and E. Vogt (Plenum Press, New York, 1971)

26) B. M. Preedom and B. H. Wildenthal, Phys. Rev. C6 (1972) 1633

27) M. Conze, H. Feldmeier and P. Manakos, Phys. Lett. 43B (1973) 101

28) T. T. S. Kuo, Nucl. Phys. A103 (1967) 71

29) T. T. S. Kuo and G. E. Brown, Nucl. Phys. 85 (1966) 40

30) J. B. McGrory, Proc. Int. Conf. on nuclear physics, Munich (1973)

31) R. R. Whitehead and A. Watt, Phys. Lett. 413 (1972) 7

32) M. Harvey, Phys. Lett. 40B (1972) 77

33) H. A. Jahn and H. van Wieringen, Proc. Roy. Soc. A209 (1951) 502

34) J. P. Elliott, J. Hope and H. A. Jahn, Proc. Roy. Soc. A246 (1953) 241

35) A. Bohr and B. R. Mottelson, Nuclear structure, vol. 1 (Benjamin, New York, 1969) p. 114 